\title{
Discontinuous Galerkin Method for Material Flow Problems
}

\author{
Simone Göttlich and Patrick Schindler \\ Department of Mathematics, University of Mannheim, 68131 Mannheim, Germany \\ Correspondence should be addressed to Simone Göttlich; goettlich@math.uni-mannheim.de
}

Received 11 August 2015; Accepted 30 September 2015

Academic Editor: Yuming Qin

Copyright (C) 2015 S. Göttlich and P. Schindler. This is an open access article distributed under the Creative Commons Attribution License, which permits unrestricted use, distribution, and reproduction in any medium, provided the original work is properly cited.

For the simulation of material flow problems based on two-dimensional hyperbolic partial differential equations different numerical methods can be applied. Compared to the widely used finite volume schemes we present an alternative approach, namely, the discontinuous Galerkin method, and explain how this method works within this framework. An extended numerical study is carried out comparing the finite volume and the discontinuous Galerkin approach concerning the quality of solutions.

\section{Introduction}

The modeling and simulation of material flow problems is motivated by engineering plant planning [1-3]. There exist various model approaches ranging from microscopic to macroscopic equations to describe the dynamic behavior of the manufacturing system; see, for example, [4-9], for an overview. The main difference is that microscopic models rely on systems of ordinary differential equations while macroscopic models are based on conservation laws. In particular for a large number of parts macroscopic models have the computational advantage that they are independent of individual parts. Therefore, with regard to the consideration of simulation and optimal control issues, macroscopic models are a beneficial tool for computation.

Macroscopic models, or conservation laws, are used in different engineering areas, for example, traffic flow [10], manufacturing systems [11], and crowd and evacuation dynamics $[12,13]$. In the case of material flow problems, active research is recently done on a theoretical level [14] or on numerical solution algorithms $[5,15,16]$.

In this work, we are concerned with a two-dimensional nonlocal hyperbolic conservation law that has been originally introduced [5]. This model has been validated against real data measurements and also provides reliable estimates on material flow and throughput rates in manufacturing system. It is even possible to rigorously derive such macroscopic models from microscopic ones via kinetic models; see [8, $9,16]$. From a numerical point of view, solution methods based on finite volume discretizations are an appropriate way to simulate material flow systems; see $[5,15]$. However, due to the underlying geometry of the problem discontinuous Galerkin methods represent an alternative approach. This has been successfully shown in [17-23] for similar structured problems in one dimension. Our intention is now to derive a discontinuous Galerkin method in two space dimensions to solve the nonlocal hyperbolic conservation law from [5].

The paper is structured as follows. A short introduction of the macroscopic model taken from [5] is mentioned in Section 2. In Section 3 we discuss two numerical solution approaches for the macroscopic models. In detail, we shortly repeat a finite volume method with dimensional splitting and introduce a discontinuous Galerkin approach. Finally, numerical results are shown in Section 4. In particular, we qualitatively compare the different presented numerical methods.

\section{Material Flow Modeling with Conservation Laws}

We consider a conveyor belt with the given geometry presented in Figure 1 in two space dimensions, where an obstacle with angle $\alpha$ in the middle of the belt is used to redirect parts. A large number of cylindrical-shaped parts are transported from the left to the right. Once the parts interact with the obstacle, queuing effects will occur. We furthermore assume that parts never move out of the $x_{1}, x_{2}$ plane. 


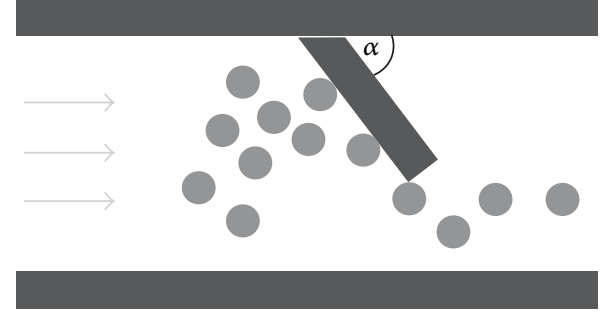

(a)

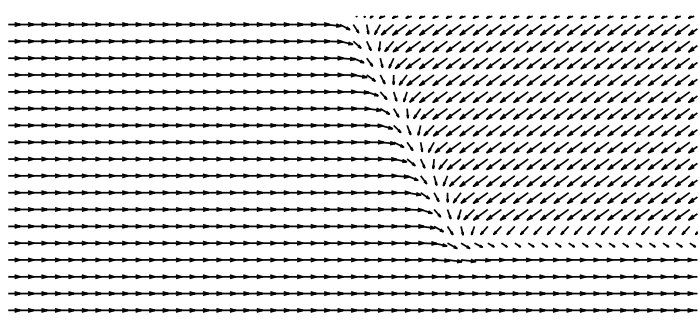

(b)

FIgURE 1: (a) Geometry of the conveyor belt. (b) Static velocity field used for numerical simulations.

We briefly recall the conservation law for the evolution of the part density $\rho$ derived in [5]. The governing equations in two space dimensions, that is, $\mathbf{x} \in \mathbb{R}^{2}$, are then

$$
\begin{aligned}
& \partial_{t} \rho+\nabla \cdot\left(\rho\left(\mathbf{v}^{\text {dyn }}(\rho)+\mathbf{v}^{\text {stat }}(\mathbf{x})\right)\right)=0, \\
& \mathbf{v}^{\text {dyn }}(\rho)=H\left(\rho-\rho_{\max }\right) \cdot \mathbf{I}(\rho), \\
& \mathbf{I}(\rho)=-\epsilon \frac{\nabla(\eta * \rho)}{\sqrt{1+\|\nabla(\eta * \rho)\|_{2}^{2}}}, \\
& \rho(\mathbf{x}, 0)=\rho_{0}(\mathbf{x}), \quad \mathbf{x} \in \mathbb{R}^{2},
\end{aligned}
$$

where $\rho=\rho(\mathbf{x}, t), H$ denotes the common Heaviside function that is zero for negative arguments and $\rho_{\max }$ the fixed maximal density. The flux function is often referred to as $\mathscr{F}(\rho)=\left(\rho\left(\mathbf{v}^{\text {dyn }}(\rho)+\mathbf{v}^{\text {stat }}(\mathbf{x})\right)\right)$.

Equation (1a) describes the evolution of the initial part density (1d) depending on velocity field composed of the dynamic velocity field $\mathbf{v}^{\mathrm{dyn}}(\rho)$ and the time-independent velocity field $\mathbf{v}^{\text {stat }}(\mathbf{x})$. The field $\mathbf{v}^{\text {stat }}(\mathbf{x})$ is induced by the movement of the conveyor belt. For the numerical simulations in Sections 3 and 4 we will use the velocity field $\mathbf{v}^{\text {stat }}(\mathbf{x})$ as indicated in Figure 1, where the right picture represents a smoothed version of the velocity field. The latter is necessary to avoid problems of well-posedness and stability in the numerical simulations. Note that all angles between 0 and 90 degrees are feasible and that the smoothing is independent of the angle that is considered.

In contrast, the dynamic component $\mathbf{v}^{\mathrm{dyn}}(\rho)$ in (1c) determines the movement of colliding parts, especially in the case they hit the obstacle. Since colliding parts do not penetrate each other, the density could not be larger than the density of a close-packing of parts $\rho_{\max }$. Therefore, we have to prevent situations, where $\rho>\rho_{\max }$ for $\rho_{0}\left(x_{1}, x_{2}\right)<\rho_{\max }$ in a certain time $t>0$. To activate the density dependent velocity $\mathbf{v}^{\mathrm{dyn}}(\rho)$, the Heaviside function in (1b) is introduced. If $\rho>\rho_{\max }$, that is, $H\left(\rho-\rho_{\max }\right)=1$, the density dependent velocity is active and inactive otherwise. Hence, the velocity field $\mathbf{v}^{\text {dyn }}(\rho)$ disperses clouds with $\rho>\rho_{\max }$ and parts are pushed into a direction with lower density.

The nonlocal operator $\mathbf{I}(\rho)$ in (1c) is controllable with the constant parameter $\epsilon>0$. Here, the negative gradient field is the steepest descent of the convolution $\eta * \rho$, where $\eta$ is a sufficiently smooth mollifier or smoothing function, for example, a Gaussian. So the density dependent force term $\mathbf{I}(\rho)$ will only act in a small neighborhood of the boundary of a congested region.

The boundary conditions of (1a), (1b), (1c), and (1d) are imposed by the geometry of the belt (cf. Figure 1). We distinguish between solid boundaries (thick black lines), where free slip conditions are used, and the inflow region at the left boundary, where homogeneous Dirichlet conditions are applied.

\section{Numerical Methods}

Now we present suitable numerical methods to solve the conservation law (1a), (1b), (1c), and (1d). The first approach is based on a one-dimensional finite volume method which is extended into a two-dimensional problem solver by dimensional splitting [5]. The other approach is a discontinuous Galerkin method which is useful to compute accurate solutions on complex geometries. Since the finite volume method is validated against real data (cf. [5]) we will use the results of this approach as a benchmark solution in Section 4 to test the performance of the discontinuous Galerkin method.

For both simulation approaches, we assume that the discontinuous flux function in (1a) can be rewritten and approximated by

$$
\begin{aligned}
\mathscr{F}(\rho) & =\left(\mathscr{F}_{1}(\rho, x), \mathscr{F}_{2}(\rho, x)\right)^{T} \\
& =\left(\rho\left(v_{1}^{\text {stat }}+v_{1}^{\text {dyn }}\right), \rho\left(v_{2}^{\text {stat }}+v_{2}^{\text {dyn }}\right)\right)^{T}, \\
v_{d}^{\text {dyn }}(\rho) & =\widetilde{H}\left(\rho-\rho_{\max }\right) I_{d}(\rho), \quad d=1,2,
\end{aligned}
$$

where $\widetilde{H}$ is a smoothed version of the Heaviside function; that is,

$$
\widetilde{H}(u)=\frac{1}{\pi} \arctan (\gamma u)+\frac{1}{2}, \quad \gamma>0 .
$$

3.1. Finite Volume Approach with Dimensional Splitting. The following procedure is based on a finite volume method with dimensional splitting; see [24]. We use discretization of the two-dimensional spatial domain in rectangular cells where each cell is identified by the indices $i, j$. The center of a cell $i, j$ is located at $\mathbf{x}_{i, j}=\left(x_{1, i}, x_{2, j}\right)^{T}$. The lengths of the cells are given by the spatial step sizes $\Delta x_{1}, \Delta x_{2}$ and the time $t$ is 
discretized by step size $\Delta t$. We use the following space and time grid:

$$
\begin{gathered}
x_{1, i}=i \Delta x_{1}, \quad i=1, \ldots, N_{x_{1}}, \\
x_{2, j}=j \Delta x_{2}, \quad j=1, \ldots, N_{x_{2}}, \\
t_{k}=k \Delta t, \quad k=1, \ldots, N_{t}
\end{gathered}
$$

with cells $Q_{i, j}=\left[x_{1, i-1 / 2}, x_{1, i+1 / 2}\right] \times\left[x_{2, j-1 / 2}, x_{2, j+1 / 2}\right]$. Additionally, grid constants are given by $\lambda_{d}=\Delta t / \Delta x_{d}$ for $d=1,2$. The density $\rho$ is defined as a step function

$$
\rho\left(\mathbf{x}, t_{k}\right)=\rho_{i, j}^{k} \in \mathbb{R} \quad \text { for } \mathbf{x} \in Q_{i, j} .
$$

Dimensional splitting is applied to split the original twodimensional problem (1a), (1b), (1c), and (1d) into a sequence of one-dimensional problems. That means, for our purpose, the flux $\rho\left(\mathbf{v}^{\text {dyn }}(\rho)+\mathbf{v}^{\text {stat }}(\mathbf{x})\right)$ used in the numerical simulation is split in each dimension according to (2a). Applying a Godunov-type scheme, the numerical flux in one dimension, that is, $d=1$, at points $\mathbf{x}_{i+1 / 2, j}$ and $t_{k}$, is a modified Roe flux combined with the nonlocal term $\mathbf{I}(\rho)=\left(I_{1}(\rho), I_{2}(\rho)\right)^{T}$ :

$$
\begin{aligned}
& F_{1}\left(\rho, \rho_{i, j}^{k}, \rho_{i+1, j}^{k}, \mathbf{x}_{i+1 / 2, j}\right) \\
& = \begin{cases}\rho_{i, j}^{k} \widetilde{H}\left(\rho_{i, j}^{k}-\rho_{\max }\right) I_{1}(\rho)\left(\mathbf{x}_{i+1 / 2, j}\right), & I_{1}(\rho)\left(\mathbf{x}_{i+1 / 2, j}\right) \geq 0 \\
\rho_{i+1, j}^{k} \widetilde{H}\left(\rho_{i+1, j}^{k}-\rho_{\max }\right) I_{1}(\rho)\left(\mathbf{x}_{i+1 / 2, j}\right), & I_{1}(\rho)\left(\mathbf{x}_{i+1 / 2, j}\right) \leq 0 .\end{cases}
\end{aligned}
$$

For $d=2$ at points $\mathbf{x}_{i, j+1 / 2}$ and time $t_{k}$ the flux $F_{2}\left(\rho, \rho_{i j}^{k}, \rho_{i, j+1}^{k}\right.$, $\left.\mathbf{x}_{i, j+1 / 2}\right)$ is determined analogously. The term $I_{1}(\rho)$ or $I_{2}(\rho)$, respectively, including the convolution is evaluated as follows:

$$
\begin{aligned}
& I_{1}(\rho)\left(\mathbf{x}_{i+1 / 2, j}\right)=-\epsilon \frac{D_{x_{1}} \rho_{i, j}}{\sqrt{1+\left(D_{x_{1}} \rho_{i, j}\right)^{2}+\left(D_{x_{2}} \rho_{i, j}\right)^{2}}}, \\
& I_{2}(\rho)\left(\mathbf{x}_{i, j+1 / 2}\right)=-\epsilon \frac{D_{x_{2}} \rho_{i, j}}{\sqrt{1+\left(D_{x_{1}} \rho_{i, j}\right)^{2}+\left(D_{x_{2}} \rho_{i, j}\right)^{2}}},
\end{aligned}
$$

where

$$
\begin{aligned}
& D_{x_{1}} \rho_{i, j}:=\sum_{p, q} \rho_{p, q}^{k} \cdot \int_{Q_{p+1 / 2, q}} \partial_{x_{1}} \eta(\boldsymbol{\tau}) d \boldsymbol{\tau}, \\
& D_{x_{2}} \rho_{i, j}:=\sum_{p, q} \rho_{p, q}^{k} \cdot \int_{Q_{p, q+1 / 2}} \partial_{x_{2}} \eta(\boldsymbol{\tau}) d \boldsymbol{\tau} .
\end{aligned}
$$

Furthermore, the static flux in $x_{1}$-direction is

$$
\begin{aligned}
& G_{1}\left(\rho_{i, j}^{k}, \rho_{i+1, j}^{k}, \mathbf{v}_{i+1 / 2, j}^{\text {stat }}\right) \\
& \quad= \begin{cases}\rho_{i, j}^{k} v_{1, i+1 / 2, j}^{\text {stat }}, & v_{1, i+1 / 2, j}^{\text {stat }} \geq 0, \\
\rho_{i+1, j}^{k} v_{1, i+1 / 2, j}^{\text {stat }}, & v_{1, i+1 / 2, j}^{\text {stat }} \leq 0,\end{cases}
\end{aligned}
$$

where the discretized static velocity field is given by

$$
\mathbf{v}_{i+1 / 2, j}^{\text {stat }}:=\left(v_{1, i+1 / 2, j}^{\text {stat }}, v_{2, i+1 / 2, j}^{\text {stat }}\right)^{T}:=\mathbf{v}^{\text {stat }}\left(\mathbf{x}_{i+1 / 2, j}\right) .
$$

Again, the flux in $x_{2}$-direction $G_{2}\left(\rho_{i, j}^{k}, \rho_{i, j+1}^{k}, \mathbf{v}_{i, j+1 / 2}^{\text {stat }}\right)$ is defined analogously.

Summarizing, we have to solve the coupled scheme

$$
\begin{aligned}
\tilde{\rho}_{i, j}^{k}= & \rho_{i, j}^{k}-\lambda_{1}\left[F_{1}^{+}-F_{1}^{-}\right], \\
F_{1}^{+}= & F_{1}\left(\rho, \rho_{i, j}^{k}, \rho_{i+1, j}^{k}, \mathbf{x}_{i+1 / 2, j}\right) \\
& +G_{1}\left(\rho_{i, j}^{k}, \rho_{i+1, j}^{k}, \mathbf{v}_{i+1 / 2, j}^{\text {stat }}\right), \\
F_{1}^{-}= & F_{1}\left(\rho, \rho_{i-1, j}^{k}, \rho_{i, j}^{k}, \mathbf{x}_{i-1 / 2, j}\right) \\
& +G_{1}\left(\rho_{i-1, j}^{k}, \rho_{i, j}^{k}, \mathbf{v}_{i-1 / 2, j}^{\text {stat }}\right), \\
\rho_{i, j}^{k+1}= & \tilde{\rho}_{i, j}^{k}-\lambda_{2}\left[F_{2}^{+}-F_{2}^{-}\right], \\
F_{2}^{+}= & F_{2}\left(\rho, \widetilde{\rho}_{i, j}^{k}, \widetilde{\rho}_{i, j+1}^{k}, \mathbf{x}_{i, j+1 / 2}\right) \\
& +G_{2}\left(\widetilde{\rho}_{i, j}^{k}, \widetilde{\rho}_{i, j+1}^{k}, \mathbf{v}_{i, j+1 / 2}^{\text {stat }}\right), \\
F_{2}^{-}= & F_{2}\left(\rho, \widetilde{\rho}_{i, j-1}^{k}, \widetilde{\rho}_{i, j}^{k}, \mathbf{x}_{i, j-1 / 2}\right) \\
& +G_{2}\left(\widetilde{\rho}_{i, j-1}^{k}, \widetilde{\rho}_{i, j}^{k}, \mathbf{v}_{i, j-1 / 2}^{\text {stat }}\right)
\end{aligned}
$$

under the stability condition (also known as CFL condition):

$$
\frac{\Delta t}{\Delta x_{d}} \max _{\rho}\left\|\frac{\partial}{\partial \rho}\left[\rho\left(\mathbf{v}^{\mathrm{d} y \mathrm{n}}(\rho)+\mathbf{v}^{\mathrm{stat}}(\mathbf{x})\right)\right]\right\|_{\infty} \leq 1,
$$

$$
\text { for } d=1,2
$$

for the smoothed Heaviside function (3). For more details and a detailed information on the algorithm we refer to [5].

3.2. Discontinuous Galerkin Methods. We now present an alternative approach to solve (1a), (1b), (1c), and (1d). Discontinuous Galerkin methods (DG methods) play an important role in finding approximations of many physical applications based on hyperbolic partial differential equations. For example, popular applications are found in gas dynamics, compressible and incompressible flows, chemical transports, granular flows, and more. We refer to [25-27] for a short overview. These methods have some interesting benefits; for example, they preserve the flexibility of finite elements in handling complicated geometries and they yield very accurate approximations. As already seen, finite volume methods use constant cell averages. In consideration of upwinding methods, this leads to artificial numerical diffusion which can influence the approximation quality. To avoid this drawback and for further investigations on optimal control issues, we consider other approximation tools such as the discontinuous Galerkin method.

In the following derivation, we assume that the flux function $\mathscr{F}(\rho)$ is approximated by polynomials. To ensure numerical stability of the DG method, we need to work again with a continuous flux function as already stated in (2a) and (3). The presentation of the DG method follows the ideas drawn in [18-21]. 


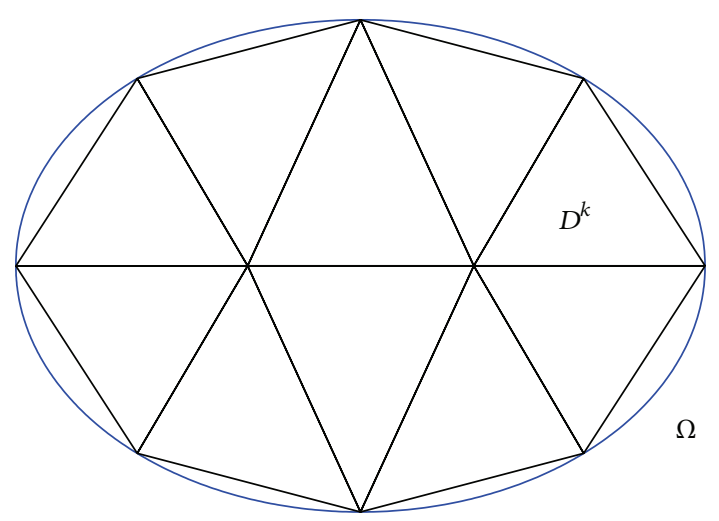

FIGURE 2: A finite element discretization (triangulation) of a domain $\Omega$ (ellipse).

\subsubsection{Space Integration. We consider a finite element dis-} cretization of the spatial domain $\Omega \simeq \Omega_{h}=\dot{\cup}_{k=1}^{K} D^{k}$, where $\Omega_{h}$ is a disjoint union of triangle elements $D^{k}$. Also, we assume that the position of each vertices of $D^{k}$ can only coincide with other vertices of neighboring triangle elements. An example of such finite element discretization or triangulation is given in Figure 2. Note that $h$ estimates the "size" of all triangle elements $D^{k}$. In this work, $h$ denotes the length of the largest triangle edge of all elements $D^{k}$.

Let $V=L^{2}\left(\Omega, \mathbb{R}^{+}\right)$be the solution space and let the approximate space $V_{h} \subset V$ be defined by

$$
V_{h}:=\left\{v \in V:\left.v\right|_{D^{k}} \in P^{N}, k=1, \ldots, K\right\},
$$

where $P^{N}$ is the space of the polynomials of degree $N$. By definition the solutions $v$ are discontinuous at the triangle interfaces. For the scheme we characterize all elements $v \in V_{h}$ by a nodal basis. In this presentation, a nodal basis is a special case of a polynomial basis. Note that a two-dimensional polynomial has

$$
N_{p}:=\frac{(N+1)(N+2)}{2}
$$

degrees of freedom for choosing the coefficients. All polynomials $\left.v\right|_{D^{k}}$, restricted to a triangle-shaped domain $D^{k}$, are constructible by nodal basis functions $\ell_{i}^{k}(\mathbf{x}) \in P^{N}$ with

$$
\ell_{i}^{k}\left(\mathbf{x}_{j}^{k}\right)=\left\{\begin{array}{ll}
1, & i=j, \\
0, & i \neq j,
\end{array} \quad \forall i, j=1, \ldots, N_{p}\right.
$$

where $\mathbf{x}_{j}^{k} \in D^{k}$ are nodal points on the finite element $k$. The polynomials $\ell_{i}^{k}(\mathbf{x})$ are called Lagrangian basis functions. The nodal points $\mathbf{x}_{i}^{k}$ for $i=1, \ldots, N_{p}$ are distributed on each triangle element $D^{k}$ as, respectively, shown in Figure 3.

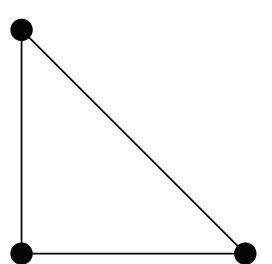

(a) $N=1$

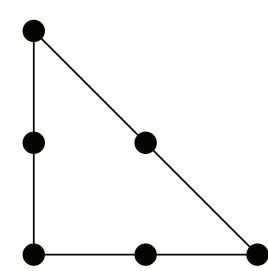

(b) $N=2$

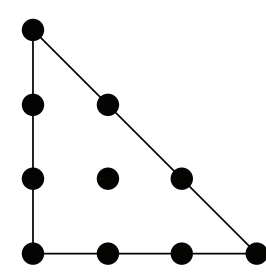

(c) $N=3$
FIGURE 3: Nodal points of the basis for linear, quadratic, and cubic triangle elements $D^{k}$.

An approximation of the solution (1a), (1b), (1c), and (1d) is given by an element of $V_{h}$; that is,

$$
\begin{aligned}
\rho_{h}^{k}(\mathbf{x}, t) & :=\sum_{i=1}^{N_{p}} \rho_{i}^{k}(t) \ell_{i}^{k}(\mathbf{x}), \\
\mathscr{F}_{h}^{k}(\mathbf{x}, t) & :=\sum_{i=1}^{N_{p}} \mathscr{F}\left(\rho_{i}^{k}(t)\right) \ell_{i}^{k}(\mathbf{x}),
\end{aligned}
$$

$$
\forall \mathbf{x} \in D^{k} .
$$

The functions $\rho_{i}^{k}(t)$ are unknowns and characterize the solution $\rho_{h}^{k}$ at time $t$. We distinguish that the approximations $\rho_{h}^{k}$ and the flux $\mathscr{F}_{h}^{k}$ fulfill (1a), (1b), (1c), and (1d) in an arbitrary way; that is,

$$
\partial_{t} \rho_{h}^{k}(\mathbf{x}, t)+\nabla \cdot \mathscr{F}_{h}^{k}(\mathbf{x}, t)=\mathscr{R}_{h}^{k}(\mathbf{x}, t), \quad \forall \mathbf{x} \in D^{k},
$$

where $\mathscr{R}_{h}^{k}(\mathbf{x}, t)$ is the residual. Generally, the approximation $\rho_{h}^{k}$ does not fulfill (1a), (1b), (1c), and (1d) exactly and the residual is not zero in all cases. Furthermore, we must decide in which sense the residual should vanish. Therefore, we choose a test function $\phi(\mathbf{x}) \in V_{h}$ that is representable as

$$
\phi_{h}^{k}(\mathbf{x}):=\sum_{i=1}^{N_{p}} \phi_{i}^{k} \ell_{i}^{k}(\mathbf{x}), \quad \forall \mathbf{x} \in D^{k} .
$$

We now require that the residual is orthogonal to all test functions in $V_{h}$; that is,

$$
\int_{D^{k}} \mathscr{R}_{h}^{k}(\mathbf{x}, t) \phi_{h}^{k}(\mathbf{x}) d \mathbf{x}=0 .
$$

This is true if and only if

$$
\int_{D^{k}} \mathscr{R}_{h}^{k}(\mathbf{x}, t) \ell_{j}^{k}(\mathbf{x}) d \mathbf{x}=0, \quad \forall j=1, \ldots, N_{p}
$$

holds. Thus, we obtain

$$
\int_{D^{k}}\left(\partial_{t} \rho_{h}^{k}(\mathbf{x}, t)+\nabla \cdot \mathscr{F}_{h}^{k}(\mathbf{x}, t)\right) \ell_{j}^{k}(\mathbf{x}) d \mathbf{x}=0 .
$$

Integrating (21) by parts yields

$$
\begin{aligned}
& \int_{D^{k}} \frac{\partial \rho_{h}^{k}(\mathbf{x}, t)}{\partial t} \ell_{j}^{k}(\mathbf{x})-\mathscr{F}_{h}^{k}(\mathbf{x}, t) \cdot \nabla \ell_{j}^{k}(\mathbf{x}) d \mathbf{x} \\
&=-\int_{\partial D^{k}} \mathbf{n} \cdot \mathscr{F}_{h}^{k}(\mathbf{x}, t) \ell_{j}^{k}(\mathbf{x}) d \mathbf{x} \quad \forall j=1, \ldots, N_{p},
\end{aligned}
$$




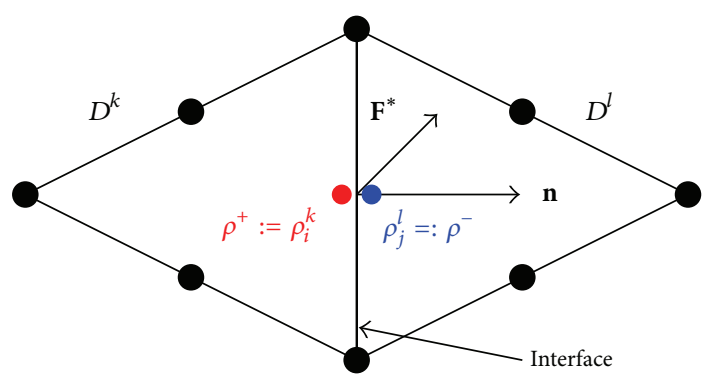

FIgURE 4: Interface of two neighboring triangles $D^{k}$ and $D^{l}$. The position of the nodal points $x_{i}^{k}$ (red) and $x_{j}^{l}$ (blue) coincides; that is, $x_{i}^{k}=x_{j}^{l}$. The interior and exterior densities $\rho^{+}$and $\rho^{-}$define the numerical flux $\mathbf{F}^{*}$ at the transition point $x_{i}^{k}=x_{j}^{l}$.

where $\mathbf{n}$ represents the local outward pointing normal. The solution at the interfaces between triangle elements is multiply-defined. At this moment, we have a lack of conditions on the local solution and the test functions. Therefore, we need here a correct combination of solutions to reduce the degrees of freedom. We select a numerical flux $\mathbf{F}^{*}$ for the fluxes at the triangle interfaces. An illustrated example is given in Figure 4. Thus, (22) leads to the local statement:

$$
\begin{gathered}
\int_{D^{k}} \frac{\partial \rho_{h}^{k}(\mathbf{x}, t)}{\partial t} \ell_{j}^{k}(\mathbf{x})-\mathscr{F}_{h}^{k}(\mathbf{x}, t) \cdot \nabla \ell_{j}^{k}(\mathbf{x}) d \mathbf{x} \\
=-\int_{\partial D^{k}} \mathbf{n} \cdot \mathbf{F}^{*} \ell_{j}^{k}(\mathbf{x}) d \mathbf{x} \quad \forall j=1, \ldots, N_{p} .
\end{gathered}
$$

In this work, especially, we choose the local Lax-Friedrichs flux for the presented DG method:

$$
\mathbf{F}^{*}\left(\rho^{+}, \rho^{-}\right)=\frac{\mathscr{F}\left(\rho^{+}\right)+\mathscr{F}\left(\rho^{-}\right)}{2}+\frac{C}{2} \mathbf{n}\left(\rho^{+}-\rho^{-}\right),
$$

where $\rho^{+}, \rho^{-}$are the interior and exterior solution value. Respectively, $C$ is the local maximum of the directional flux

$$
C=\max _{\rho \in\left[\rho^{+}, \rho^{-}\right]}\left|n_{x} \frac{\partial \mathscr{F}_{1}}{\partial \rho}+n_{y} \frac{\partial \mathscr{F}_{2}}{\partial \rho}\right| .
$$

The goal is to achieve an ODE system to obtain the quantity $\rho_{i}^{k}(t)$. We plug now (16) into (22) and we get the following statement:

$$
\begin{aligned}
\sum_{i=1}^{N_{p}} & {\left[\frac{\partial \rho_{i}^{k}(t)}{\partial t} \int_{D^{k}} \ell_{i}^{k}(\mathbf{x}) \ell_{j}^{k}(\mathbf{x}) d \mathbf{x}-\mathscr{F}\left(\rho_{i}^{k}(t)\right)\right.} \\
& \left.\cdot \int_{D^{k}} \ell_{i}^{k}(\mathbf{x}) \nabla \ell_{j}^{k}(\mathbf{x}) d \mathbf{x}\right]=-\int_{\partial D^{k}} \mathbf{n} \\
& \cdot \sum_{i=1}^{N_{p}} \mathbf{F}^{*} \ell_{i}^{k}(\mathbf{x}) \ell_{j}^{k}(\mathbf{x}) d \mathbf{x}=-\sum_{e=1}^{3} \int_{\text {interface } e} \mathbf{n}_{e} \\
& \cdot \sum_{i=1}^{N_{p}} \mathbf{F}^{*} \ell_{i}^{k}(\mathbf{x}) \ell_{j}^{k}(\mathbf{x}) d \mathbf{x},
\end{aligned}
$$

where $\mathbf{n}_{e}$ denotes the outward pointing normal of the interface $e$ of the triangle $D^{k}$. The ODE system (26) can be written into a matrix notation; that is,

$$
\begin{aligned}
\mathscr{M}^{k} & \frac{\partial \rho^{k}(t)}{\partial t}+\delta_{1}^{k} \mathscr{F}_{1}\left(\rho^{k}(t)\right)+\mathcal{S}_{2}^{k} \mathscr{F}_{2}\left(\rho^{k}(t)\right) \\
& =-\sum_{e=1}^{3} \mathscr{M}^{k, e}\left(\mathbf{n}_{e} \cdot \mathbf{F}^{*}\right),
\end{aligned}
$$

where $\rho^{k}$ is a vector of dimension $N_{p}$ containing the cell unknowns $\rho_{i}^{k}$. The local mass matrices $\mathscr{M}^{k}$ and the stiffness matrices $\delta_{1}^{k}, \delta_{2}^{k}$ are defined by

$$
\begin{aligned}
\mathscr{M}_{i, j}^{k}= & \int_{D^{k}} \ell_{i}^{k}(\mathbf{x}) \ell_{j}^{k}(\mathbf{x}) d \mathbf{x}, \\
\mathcal{S}_{d, i, j}^{k}= & \int_{D^{k}} \ell_{i}^{k}(\mathbf{x}) \partial_{x_{d}} \ell_{j}^{k}(\mathbf{x}) d \mathbf{x}, \\
d=1,2, \forall i, j=1, \ldots, N_{p}, k=1, \ldots, K, & \ell_{i, j}^{k, e}=\int_{\text {interface } e} \ell_{i}^{k}(\mathbf{x}) \ell_{j}^{k}(\mathbf{x}) d \mathbf{x}, \quad e=1,2,3 .
\end{aligned}
$$

Remark 1. The coefficient matrices $\mathscr{M}_{i, j}^{k}, \delta_{d, i, j}^{k}, \mathscr{M}_{i, j}^{k, e}$ for $d=$ 1,2 and $e=1,2,3$ depend only on the choice of the basis functions and the corresponding triangulation. Therefore, it is useful to compute these matrices once only for a complete simulation. This can be done by a preprocessing routine.

3.2.2. Discontinuous and Shock Solutions: Filtering. It is well known that nonlinear conservation laws might lead to shocks or discontinuities in solutions. However, the polynomial approximation of solutions of the DG method is not able to prescribe discontinuities so far. If we apply the previous DG method to problems with shock solutions, the following problems will occur:

(i) The appearance of artificial and persistent oscillations around the point of discontinuity.

(ii) The loss of pointwise convergence at the point of discontinuity.

This phenomenon is already known as the Gibbs phenomenon and its behavior is well understood [28].

Note that a high order polynomial basis on the elements gives a high order accuracy of the scheme for smooth solutions. However, the DG method handles discontinuities with persistent oscillations that distort the approximate solution or influence the stability properties. Therefore, we propose the following filter approach in stabilizing the computations and in reducing the oscillations.

The filter approach $[29,30]$ considers ways to recover some accuracy information hidden in the oscillatory solutions. One possibility is filtering out high frequent redundant oscillations (high order polynomials) in the solutions. 


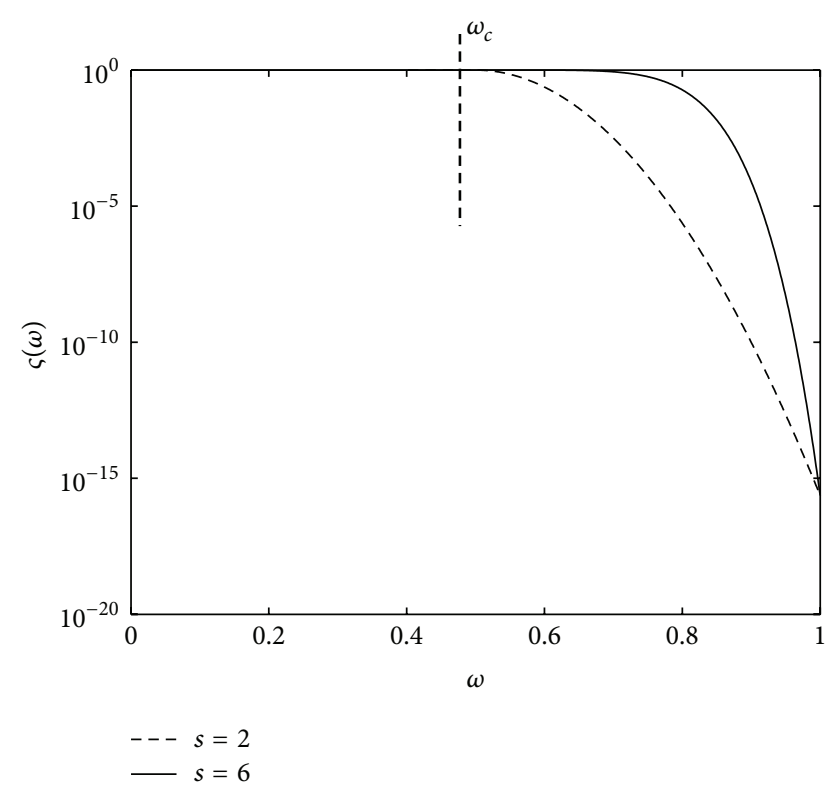

(a) $\beta=36, \omega_{c}=0.5$

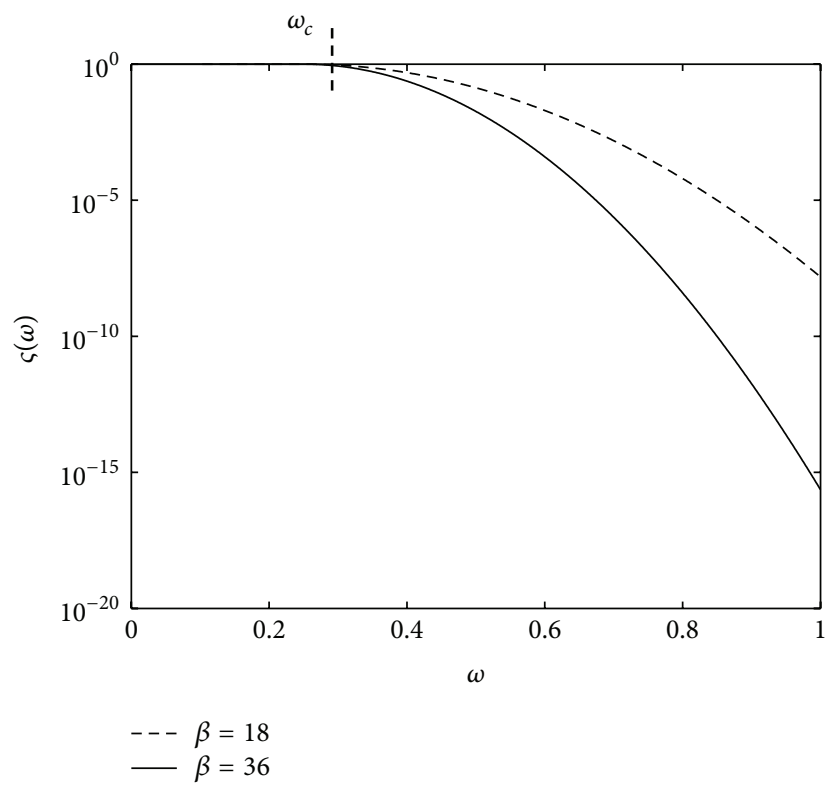

(b) $s=2, \omega_{c}=0.25$

Figure 5: Examples of how the filter function (34) varies from the three parameters: the order $s$, the cutoff $N_{c}=N \omega_{c}$, and the maximum damping parameter $\beta$.

In the following, we consider the canonical basis

$$
\begin{aligned}
\psi_{m}(\mathbf{r}) & =r_{1}^{i} r_{2}^{j}, \quad(i, j) \geq 0 ; i+j \leq N, \\
m & :=j+(N+1) i+1-\frac{i}{2}(i-1),
\end{aligned}
$$

$$
(i, j) \geq 0 ; i+j \leq N
$$

which spans the space of $\mathrm{N}$-dimensional polynomials in two variables $\mathbf{r}=\left(r_{1}, r_{2}\right)$. Additionally, the spatial variable $\mathbf{r}$ is restricted to a reference triangle $I$; that is, $\mathbf{r} \in I:=\left\{\left(r_{1}, r_{2}\right)\right.$ : $\left.r_{1}, r_{2} \geq-1, r_{1}+r_{2} \leq 0\right\}$. However, it is a complete polynomial basis and it can be orthonormalized through a Gram-Schmidt process. The resulting basis is denoted by $\widetilde{\psi}_{m}(\mathbf{r})$. The next step is to transform the basis function $\widetilde{\psi}_{m}(\mathbf{r})$ back on a triangle element $D^{k}$. This is realizable by a linear mapping $\Psi: I \rightarrow$ $D^{k}$. Thus, we obtain the basis function on $D^{k}$ by $\widetilde{\psi}_{m}^{k}(\mathbf{x}):=$ $\widetilde{\psi}\left(\Psi^{-1}(\mathbf{x})\right)$ with the property

$$
\int_{D^{k}} \widetilde{\psi}_{m}(\mathbf{x}) \widetilde{\psi}_{n}(\mathbf{x}) d \mathbf{x}=\delta_{m, n} .
$$

An approximate solution of an element $D^{k}$ is given by

$$
\rho_{h}^{k}(\mathbf{x})=\sum_{i=1}^{N_{p}} \rho_{i}^{k} \ell_{i}^{k}(\mathbf{x})=\sum_{m=1}^{N_{p}} \widetilde{\rho}_{m}^{k} \widetilde{\psi}_{m}^{k}
$$

The solution above is given in a multidimensional Lagrange polynomial basis $\ell_{i}^{k}$. Now we transform $\rho_{h}^{k}(\mathbf{x})$ into the basis consisting of $\widetilde{\psi}_{m}^{k}$. Note that the polynomial $\widetilde{\psi}_{m}^{k}$ has the degree $\operatorname{deg}\left(\widetilde{\psi}_{m}^{k}\right)=i+j$. The idea of filtering is to reduce the coefficient $\widetilde{\rho}_{m}^{k}$ of high polynomial basis elements $\widetilde{\psi}_{m}^{k}$. A popular choice is the exponential filter

$$
\varsigma(\omega)=\exp \left(-\beta \omega^{2 s}\right)
$$

to obtain the filtered expansion

$$
\rho_{h}^{k, F}(\mathbf{x})=\sum_{i, j \geq 0}^{i+j \leq N} \varsigma\left(\frac{i+j}{N}\right) \tilde{\rho}_{m}^{k} \widetilde{\psi}_{m}^{k},
$$

where the filter is characterized by the the maximum damping parameter $\beta>0$ and the order $s>0$. It is reasonable to use other filter approaches; see $[29,30]$. In this work, we use a filter of the form

$$
\varsigma(\omega)= \begin{cases}1, & 0 \leq \omega \leq \omega_{c}=\frac{N_{c}}{N}, \\ \exp \left(-\beta \frac{\left(\omega-\omega_{c}\right)}{\left(1-\omega_{c}\right)^{2 s}}\right), & \omega_{c} \leq \omega \leq 1 .\end{cases}
$$

The filter (34) is an extension of the exponential filter (32). $N_{c}$ presents a cutoff; that is, polynomials $\tilde{\rho}_{m}^{k}$ with degree $\operatorname{deg}\left(\widetilde{\rho}_{m}^{k}\right) \leq N_{c}$ are left untouched. An example of the filter (34) with different parameters is shown in Figure 5.

Since filtering usage should be used both as minimal as possible and as much as needed, this is necessary to stabilize the method, reduce oscillatory solutions, and reduce artificial viscosity.

3.2.3. Convolution Integration. In particular, the dispersive term I $(\rho)$ of (1a), (1b), (1c), and (1d) depends on the convolution of the density $\rho$ and the gradient of the mollifier $\eta$; that is,

$$
\nabla(\eta * \rho)=\left(\partial_{x_{1}} \eta * \rho, \partial_{x_{2}} \eta * \rho\right)^{T} .
$$


Hence, it is necessary to include the convolution process into the discontinuous Galerkin scheme. Without loss of generality, we consider the convolution of the approximate solution $\rho_{h} \in V_{h}$ and $\partial_{1} \eta$ in the nodal point $\mathbf{x}_{i}^{k}$ of a triangle $k$; that is,

$$
\begin{aligned}
\left(\partial_{x_{1}} \eta * \rho_{h}\right)\left(\mathbf{x}_{i}^{k}\right) & =\int_{\Omega} \eta\left(\mathbf{x}_{i}^{k}-\boldsymbol{\tau}\right) \rho_{h}(\boldsymbol{\tau}) d \boldsymbol{\tau} \\
& =\sum_{l=1}^{K} \int_{D^{l}} \eta\left(\mathbf{x}_{i}^{k}-\boldsymbol{\tau}\right) \rho_{h}^{l}(\boldsymbol{\tau}) d \boldsymbol{\tau} \\
& =\sum_{l=1}^{K} \int_{D^{l}} \eta\left(\mathbf{x}_{i}^{k}-\boldsymbol{\tau}\right) \sum_{j=1}^{N_{p}} \rho_{j}^{l} \ell_{j}^{l}(\boldsymbol{\tau}) d \boldsymbol{\tau} \\
& =\sum_{l=1}^{K} \sum_{j=1}^{N_{p}} \rho_{j}^{l} \underbrace{\int_{D^{l}} \eta\left(\mathbf{x}_{i}^{k}-\boldsymbol{\tau}\right) \ell_{j}^{l}(\boldsymbol{\tau}) d \boldsymbol{\tau}}_{:=c_{i, j}^{k, l}} \\
& =\sum_{l=1}^{K} \sum_{j=1}^{N_{p}} \rho_{j}^{l} c_{i, j}^{k, l} .
\end{aligned}
$$

The computation for the convolution of $\rho_{h} \in V_{h}$ and $\partial_{2} \eta$ works analogously.

Remark 2. Note that the weights $c_{i, j}^{k, l}$ are time independent. Therefore, $c_{i, j}^{k, l}$ can be computed once only before the simulation starts. However, the computation can result in high computational efforts for a large number of triangles $K$ and polynomial degree $N$. Under certain circumstances, it is necessary to determine and store a number of $\mathcal{O}\left(\left(N_{p} K\right)^{2}\right)$ weights to evaluate the convolution $\left(\partial_{x_{1}} \eta * \rho_{h}\right)$ for all nodal points.

3.2.4. Time Integration. The DG approximation leads to a system of $N_{p}$ ordinary differential equations (ODEs) over each element $D^{k}$. After inverting the local mass matrix $\mathscr{M}_{k}$, system (27) can be transformed in the following matrix form:

$$
\frac{d \rho^{k}(t)}{d t}=\mathscr{A}\left(\rho^{k}\right),
$$

where $\rho^{k}(t)$ is a vector of dimension $N_{p}$ containing the cell unknowns $\rho_{i}^{k} . \mathscr{A}\left(\rho^{k}\right)$ denotes the components of the right hand side of the ODE system (27) multiplied by the inverse mass matrix $\mathscr{M}_{i, j}^{k}$. The corresponding ODE system can be solved by explicit methods, for example, the forward Euler method.

As a result, the DG computation procedure is illustrated by the following steps:

(1) Computation of $\tilde{\rho}^{k}$ is given as follows:

$$
\tilde{\rho}^{k}=\rho^{k}\left(t_{n}\right)+\Delta t \mathscr{A}\left(\rho^{k}\left(t_{n}\right)\right), \quad \forall k=1, \ldots, K .
$$

(2) Reconstruction of the updated solution $\widetilde{\rho}^{k}$ is given by applying

$$
\rho^{k}\left(t_{n+1}\right)=\mathscr{U}\left(\tilde{\rho}^{k}\right), \quad \forall k=1, \ldots, K,
$$

where $\mathcal{U}$ denotes the filter process that is discussed above.

\section{Numerical Results}

Finally, we present computational results comparing the finite volume approach and the discontinuous Galerkin method presented in Section 3. In particular, we comment on the quality of solutions and analyze lane and pattern artifacts.

All computations are performed on the same platform, namely, a 3.0 GHz Dual Core computer with $8 \mathrm{~GB}$ RAM, and all algorithms are implemented in MATLAB.

4.1. Finite Volume versus Discontinuous Galerkin. First, we compare the quality of the two methods to numerically solve (1a), (1b), (1c), and (1d). The finite volume method and the discontinuous Galerkin method offer their benefits as well as drawbacks that are independently discussed in this section.

4.1.1. Macroscopic Model Settings. The field $\mathbf{v}^{\text {stat }}(\mathbf{x})$ is given by a smoothed velocity field as indicated in Figure 1(b), where the obstacle angle $\alpha$ is set to 60 degrees. We choose a smoothed version of the Heaviside function with $\gamma=25$ according to (3). The mollifier $\eta$, occurring in the operator $\mathbf{I}(\rho)$, is defined as

$$
\eta(\mathbf{x})=\frac{\sigma}{2 \pi} \exp \left(-\frac{1}{2} \sigma\|\mathbf{x}\|_{2}^{2}\right), \quad \sigma=2500
$$

In this example, the maximal density is set to $\rho_{\max }=1$. The strength of the term $\mathbf{I}(\rho)$ is selected as $\epsilon=2 v_{T}$, where the velocity of the conveyor belt is $v_{T}=0.395 \mathrm{~m} / \mathrm{s}$. Furthermore, the total time horizon is $T=7$.

4.1.2. Finite Volume Settings. The grid sizes of the finite volume approach with dimensional splitting are chosen as $\Delta x_{1}=$ $\Delta x_{2}=5 \cdot 10^{-3}, \Delta t=1.25 \cdot 10^{-3}$ in the following computation.

4.1.3. Discontinuous Galerkin Settings. The discontinuous Galerkin method uses a triangulation $\Omega_{h}$ with a maximal triangle edge length $h=0.1$. The polynomial degree of each finite element is $N=10$. ODE system (27) is solved by the explicit Euler method with a time step size $\Delta t=10^{-3}$. Thereby the filter procedure is called in each computational step of the ODE solver. The filter settings are selected as $\beta=36, s=6$, and $N_{c}=1$.

The results are shown in Figure 6. The left column shows the solution computed by the finite volume approach with splitting. The right column shows the results of the discontinuous Galerkin method. Each picture shows the density function as a gray-scaled image plot and each color specifies a density value. Thus, a dark color represents a higher density (black represents the maximal density) and vice versa. In all results, we observe that the parts are transported by the conveyor belt velocity $v_{T}$. A formation of congestion can be seen in all results.

In all plots, we recognize a weak dispersing of density (cf. Figures $6(\mathrm{~g})$ and $6(\mathrm{~h})$ ). This is caused by the term $\mathbf{v}^{\text {dyn }}=$ $\widetilde{H}\left(\rho-\rho_{\max }\right) \mathbf{I}(\rho)$. The smoothed modification $\widetilde{H}\left(\rho-\rho_{\max }\right)$ is never zero for $\rho<\rho_{\max }$. Consequently, the dispersing term $\mathbf{I}(\rho)$ is always activated and the quantity drifts apart all 


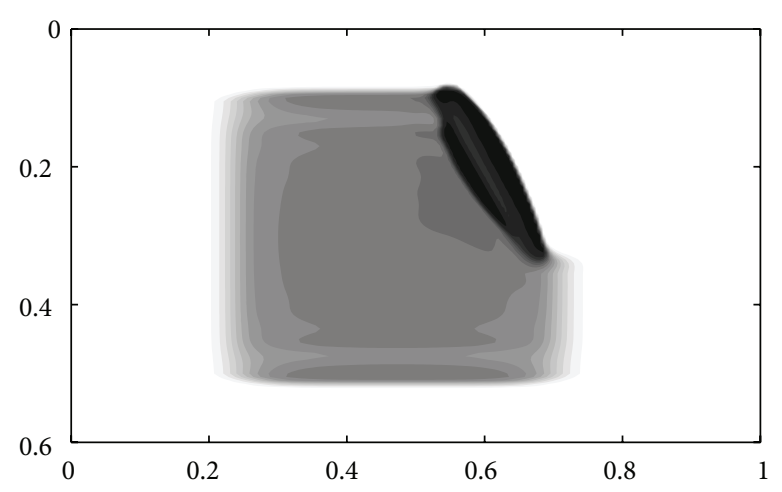

(a) Time $t=0.50$

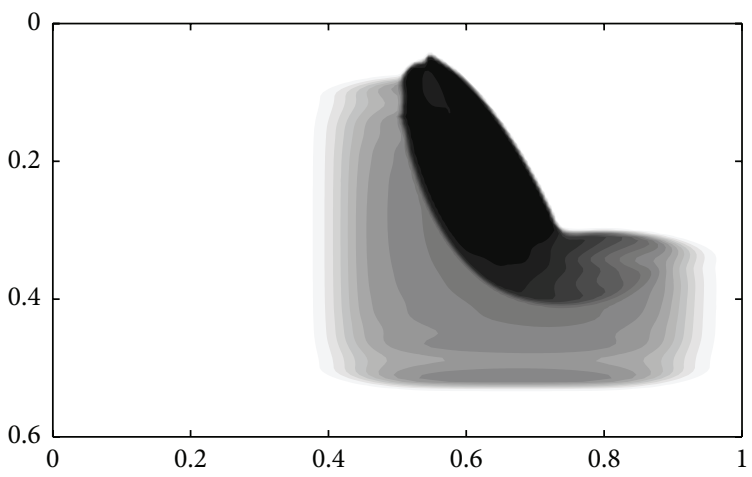

(c) Time $t=1.00$

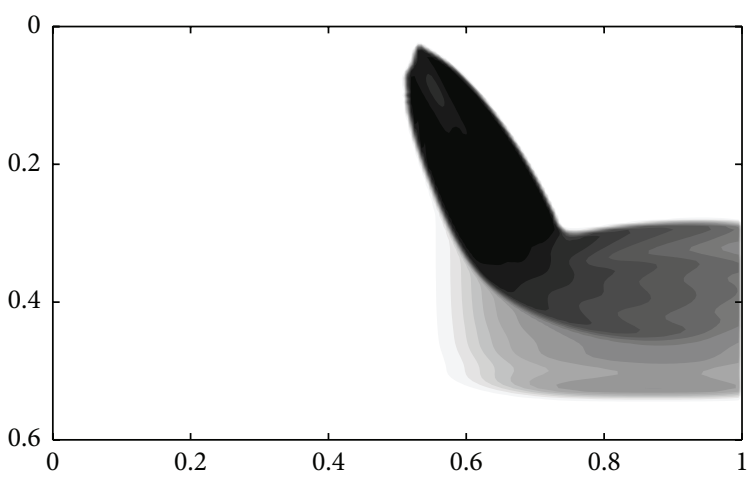

(e) Time $t=1.50$

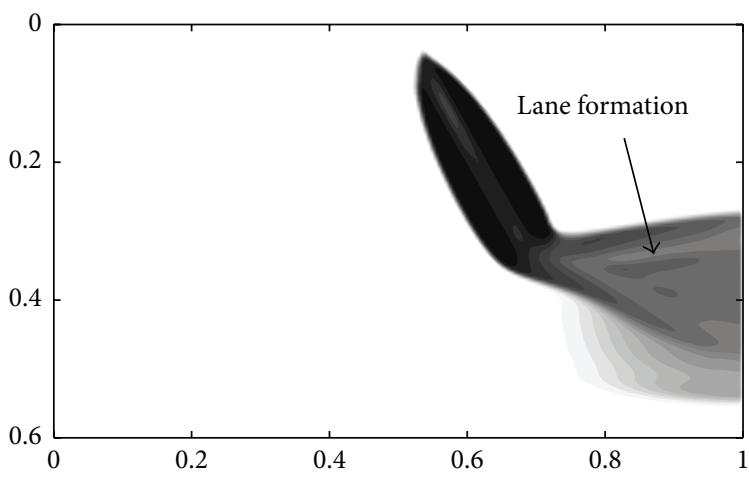

(g) Time $t=2.00$

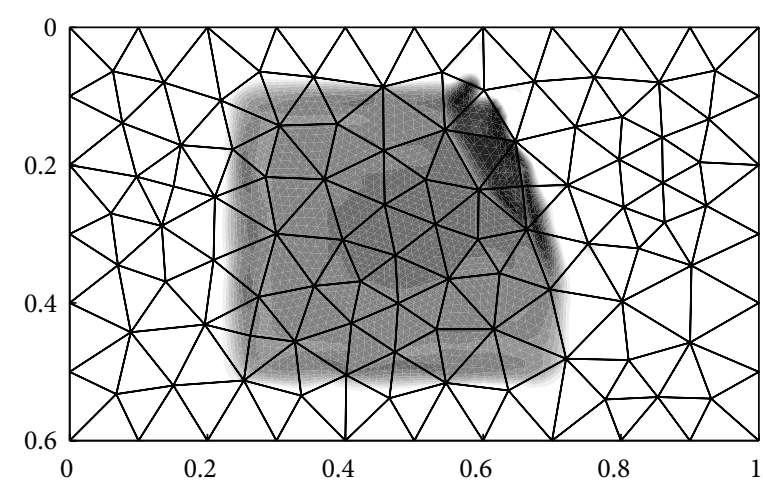

(b) Time $t=0.50$

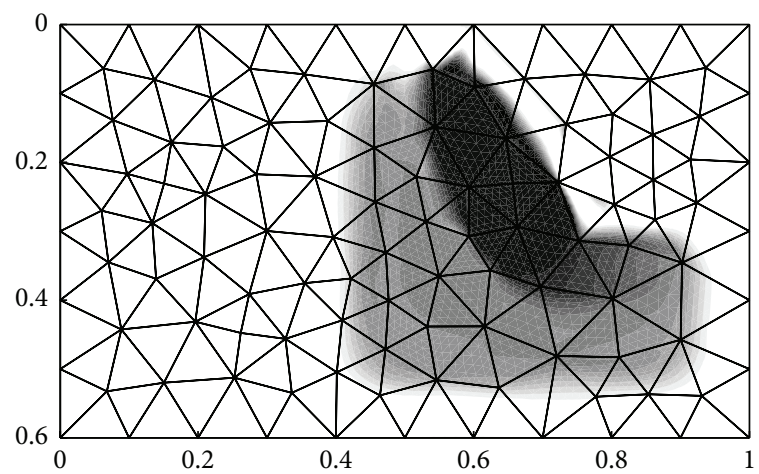

(d) Time $t=1.00$

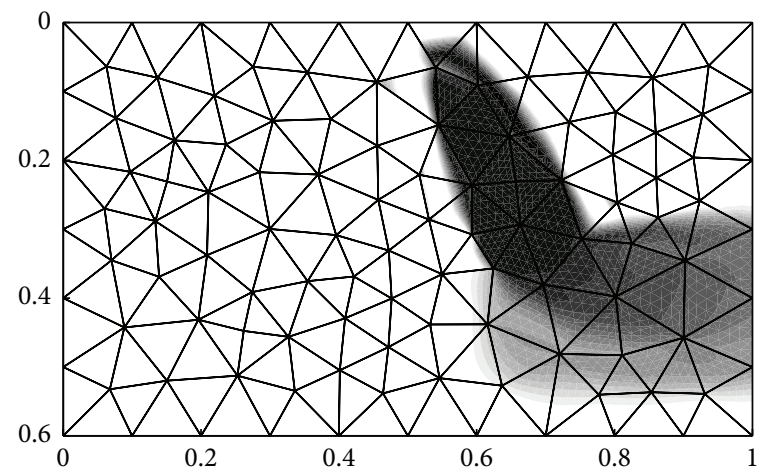

(f) Time $t=1.50$

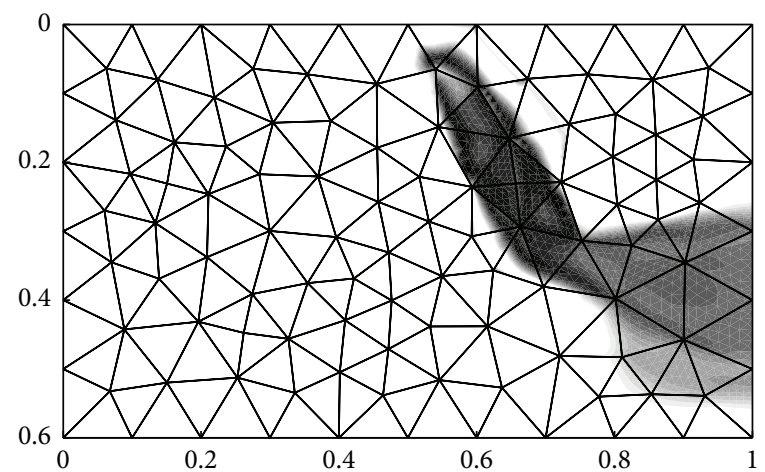

(h) Time $t=2.00$

FIGURE 6: Results of the finite volume method with splitting: $\Delta x_{1}=\Delta x_{2}=5 \cdot 10^{-3}$ (left) and results of the discontinuous Galerkin method: $h \approx 0.1, N=10$ (right). 


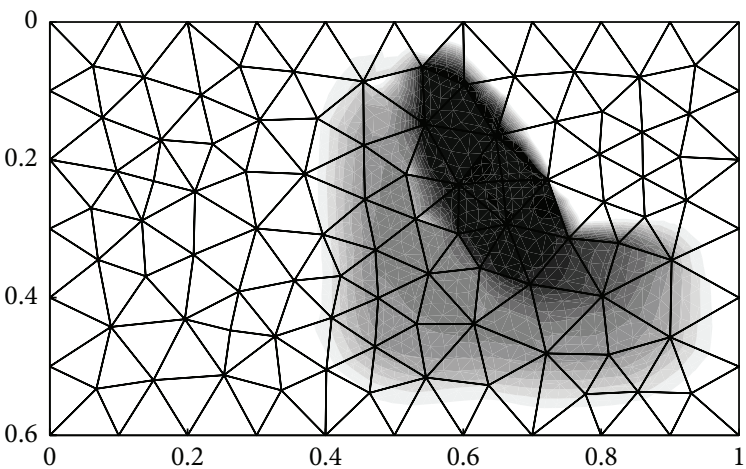

(a) $h \approx 0.1, N=5$

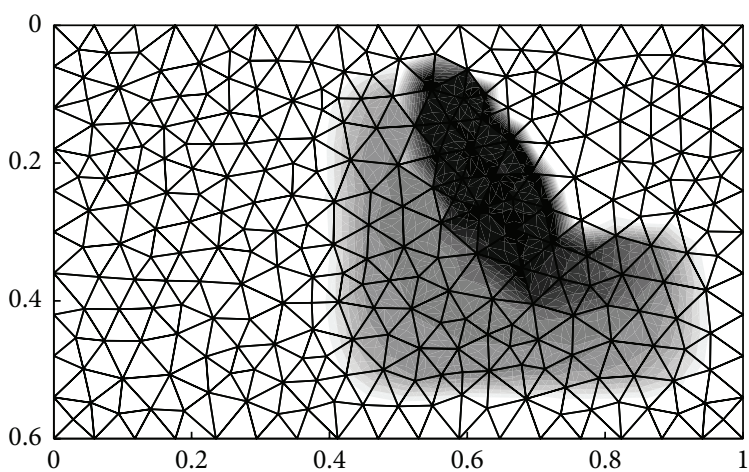

(c) $h \approx 0.06, N=3$

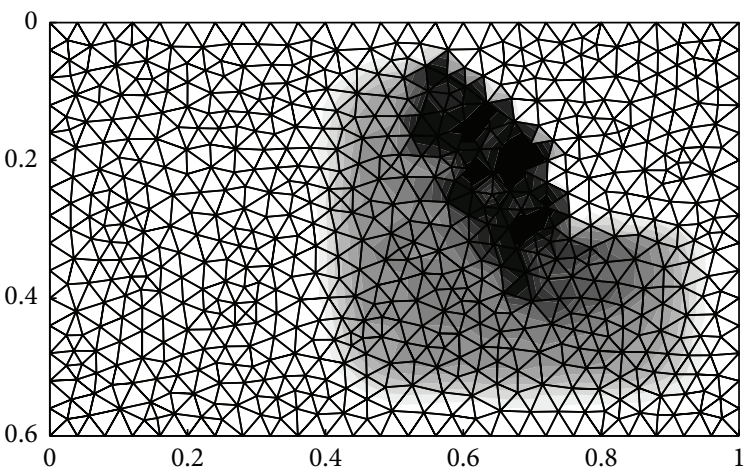

(e) $h \approx 0.04, N=1$

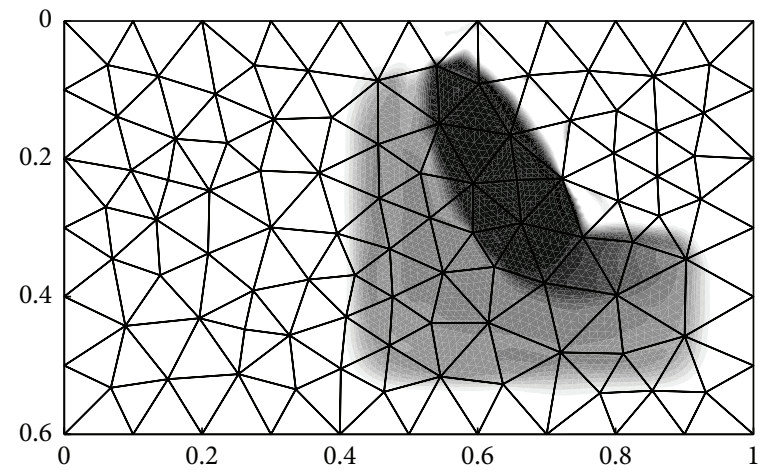

(b) $h \approx 0.1, N=11$

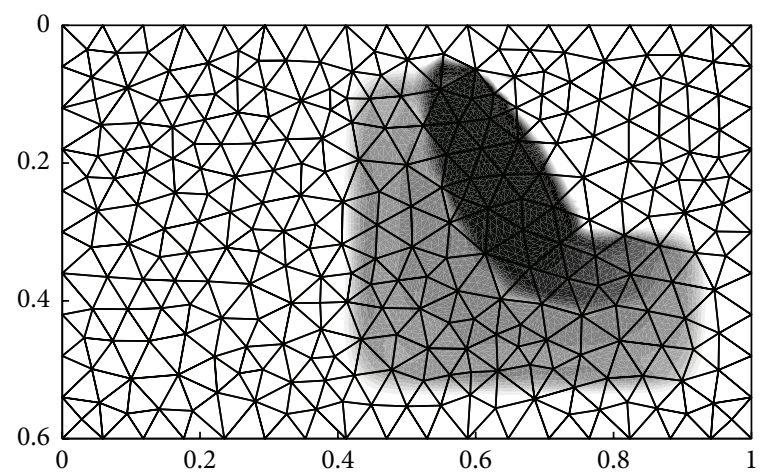

(d) $h \approx 0.06, N=7$

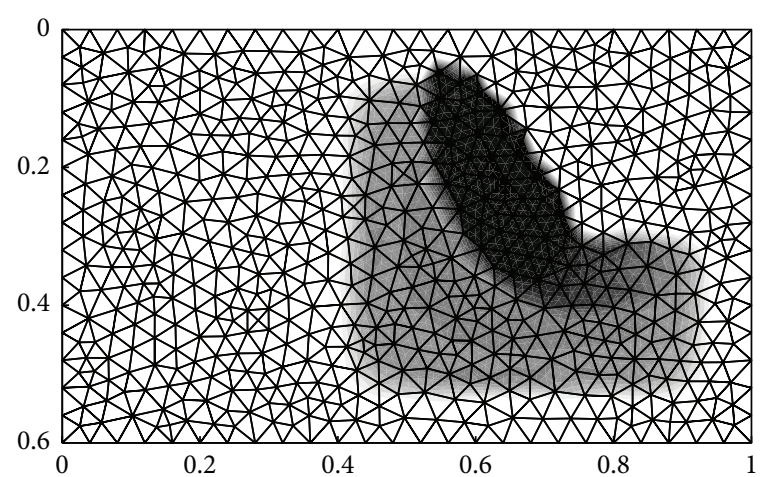

(f) $h \approx 0.04, N=3$

FiguRE 7: Results of the discontinuous Galerkin method with different triangulations $(h=0.1,0.06,0.04)$ and polynomials degrees $N$. All plots show the solution at time $t=1$.

the time. This is also true, if the quantity has no connection to the singularizer; a dispersing effect is also recognizable (see Figures 6(a) and 6(b)). Moreover, the term $\mathrm{I}(\rho)$ disperses the quantity with addition of artifacts (lane formation). Indeed, lane formations are observable, for example, in Figure 6(g). The solution of the discontinuous Galerkin method seems to be smooth and not accurate in contrast to the results of the finite volume method. This is mainly due to the fact that the DG method uses polynomials on triangle finite elements of degree $N=10$. However, polynomials are inherently smooth, and it is impossible to approximate accurate shock solutions due to the presented size of the finite elements. Indeed, the quality of the DG method can be improved by refining the triangle mesh grid. Compared to the DG method, the splitting method uses 20 times higher discretization.

The following question rises: what mesh grid sizes and what polynomial degrees are necessary to ensure good approximations due to the discontinuous Galerkin method. In the following, the previous example is computed again by the DG method with different triangulations and polynomial degrees. We test our problem on 3 different mesh grid sizes $h=0.1, h=0.06$, and $h=0.04$. The results are shown in Figure 7. For all grid sizes and polynomial degrees, the qualitative behavior of the solution is approximated quite 
TABLE 1: Computation times of the discontinuous Galerkin method (simulation process) with different grid sizes $h$ and polynomial degrees $N$. The time is measured in seconds.

\begin{tabular}{lccc}
\hline$N$ & $h=0.1$ & $h=0.06$ & $h=0.04$ \\
\hline 1 & $7.14 \mathrm{~s}$ & $12.30 \mathrm{~s}$ & $13.42 \mathrm{~s}$ \\
3 & $9.30 \mathrm{~s}$ & $18.63 \mathrm{~s}$ & $51.70 \mathrm{~s}$ \\
5 & $17.31 \mathrm{~s}$ & $46.29 \mathrm{~s}$ & - \\
7 & $30.10 \mathrm{~s}$ & $111.94 \mathrm{~s}$ & - \\
9 & $49.58 \mathrm{~s}$ & - & - \\
11 & $88.70 \mathrm{~s}$ & - & - \\
\hline
\end{tabular}

TABLE 2: Computation times in seconds for the convolution preprocessing due to the grid size $h$ and polynomial degree $N$.

\begin{tabular}{lccc}
\hline$N$ & $h=0.1$ & $h=0.06$ & $h=0.04$ \\
\hline 1 & $0.06 \mathrm{~s}$ & $1.12 \mathrm{~s}$ & $1.73 \mathrm{~s}$ \\
3 & $0.48 \mathrm{~s}$ & $3.88 \mathrm{~s}$ & $79.99 \mathrm{~s}$ \\
5 & $2.01 \mathrm{~s}$ & $60.82 \mathrm{~s}$ & - \\
7 & $5.44 \mathrm{~s}$ & $420.08 \mathrm{~s}$ & - \\
9 & $47.11 \mathrm{~s}$ & - & - \\
11 & $183.86 \mathrm{~s}$ & - & - \\
\hline
\end{tabular}

well. A finer grid or a higher polynomial degree generates more precise solutions; that is, quantity shocks and congested formations are drawn in an accurate way.

However, a rough triangulation or a low polynomial degree causes bad approximations (cf. Figure 7(e)). Compared to the other results, the congestion formation in Figure 7(e) looks quite degenerated.

The computation times of the DG method with respect to the mesh-sizes and polynomial degrees are shown in Tables 1 and 2. Furthermore, the computation times are distinguished into preprocessing time (cf. Table 2) and simulation time (cf. Table 1). Preprocessing contains the calculation of the coefficients of the convolution (see Remark 2). The simulation time contains the computation of ODE system (27) by the explicit Euler method.

The computing time required for the calculation of the finite volume approach is about $788.21 \mathrm{~s}$. Consequently, the DG method is quite faster than the finite volume approach for all presented settings. However, the computing times and the memory requirements of the DG preprocessing increase enormously since the computation of the convolution in one nodal point requires at most $N_{p} \cdot K$ coefficients. Furthermore, there are $N_{p} \cdot K$ nodal points and the convolution is evaluated twice in each dimension. Thus, it is necessary to calculate and store about $2 \cdot\left(N_{p} \cdot K\right)^{2}$ coefficients. As a consequence, the computer was not able to run the preprocessing routine successfully for small $h$ and a large $N$; for example, $N=11$ and $h=0.06$; see Table 2 .

Let us summarize. The discontinuous Galerkin method is able to approximate accurately the extended flow equations on complex geometric domains. However, the presented example consists only of a rectangle-shaped domain and it is not necessary to use methods for complex geometries (cf. regular grids). As already seen, the DG method needs a very time and memory consuming preprocessing due to the convolution. Hence, it is very expensive to apply small step sizes $h$ for computation of accurate approximations and evaluating the corresponding convergence behavior.

4.2. Lane and Pattern Formation. The macroscopic model (1a), (1b), (1c), and (1d) is based on an integral-differential equation using a convolution term in the flux function $\mathscr{F}(\rho)$. Similar models are already used for pedestrian flows [12], where certain lane or pattern artifacts are already observed. In this regard, it is not clearly understood why lane or pattern formation occurs. To investigate the phenomena of lane formation in more detail, we are motivated to study these artifacts by applying different numerical schemes.

Pedestrian models as [12] do not limit the influence of the dispersive term $\mathbf{I}(\rho)$ to a maximum density in (1a), (1b), (1c), and (1d) and therefore do not need the Heaviside function. If we additionally neglect the static velocity field $\mathbf{v}^{\text {stat }}$ in (1a), (lb), (1c), and (1d), the conservation law reduces to

$$
\begin{aligned}
\partial_{t} \rho+\nabla \cdot(\rho(\mathbf{I}(\rho))) & =0, \\
\mathbf{I}(\rho) & =-\frac{\nabla(\eta * \rho)}{\sqrt{1+\|\nabla(\eta * \rho)\|_{2}^{2}}} .
\end{aligned}
$$

In [12], lane formation was observed for the pedestrian model with smooth dispersive term, whereas this effect seems to be much less present in the above presented nonsmooth material flow model. Note that the finite volume method and the discontinuous Galerkin approach as well work with a smoothed Heaviside function. Therefore, we also observe lane artifacts in Figure 6(g).

We solve the simplified equation (41) on the spatial domain $\Omega=[-1,1]^{2}$. The initial density $\rho(\mathbf{x}, 0)$ is set to 1 for $\mathbf{x} \in[-1 / 2,1 / 2]^{2}$; otherwise $\rho(\mathbf{x}, 0)=0$. Additionally, we compute the simplified model (41) for three different mollifiers; that is, $\sigma=25,100,400$. The step sizes of the finite volume approach are $\Delta x_{1}=\Delta x_{2}=0.01$ and $\Delta t=0.005$.

The results of the finite volume approach are shown in Figure 8 . In all plots, we observe that the quantity spreads out in all directions. Figures $8(\mathrm{a})-8(\mathrm{c})$ correspond to the setting with smoothing function parameter $\sigma=25$. We recognize a squared-shaped pattern in all time series. In Figures 8(d)$8(\mathrm{f})$ and $8(\mathrm{~g})-8(\mathrm{i})$, the smoothing function parameter $\sigma=$ 100,400 is used. Here, we observe a lane formation with a circular shape. A further increase of the mollifier parameter $\sigma$ yields thinner lanes in the solution. However, we recognize the disappearance of the lanes in Figures 8(h) and 8(i). This is caused by the artificial numerical diffusion of the scheme which smears out the thin lanes in the solution.

Figure 9 shows the results of the discontinuous Galerkin method for different triangulations and polynomial degrees; however, the results are plotted for the time $t=2$ and $\sigma=100$. 


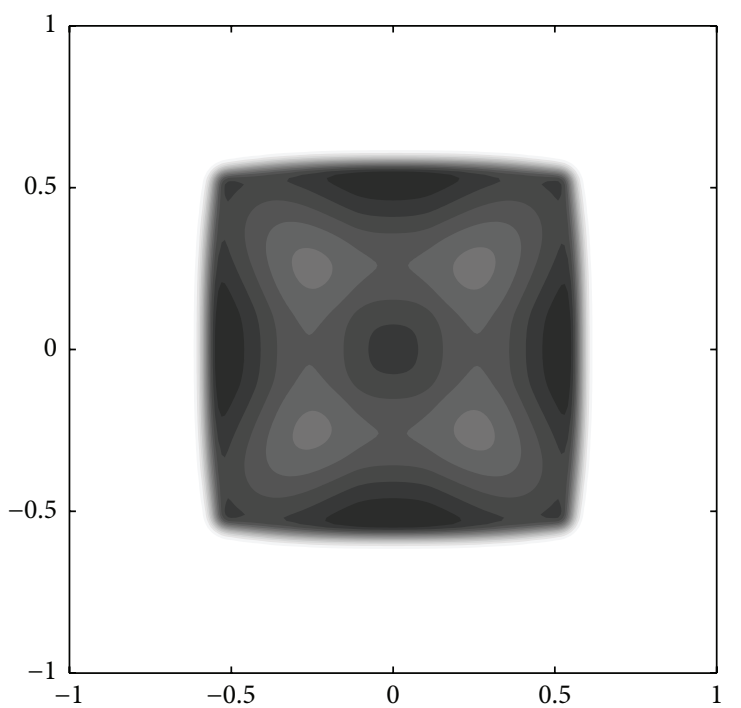

(a) Time $t=0.1, \sigma=25$

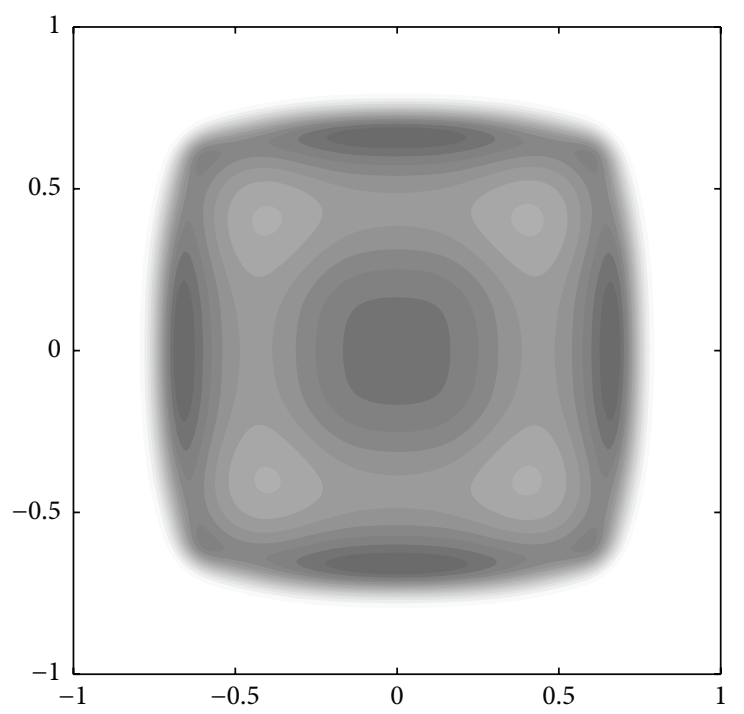

(c) Time $t=0.3, \sigma=25$

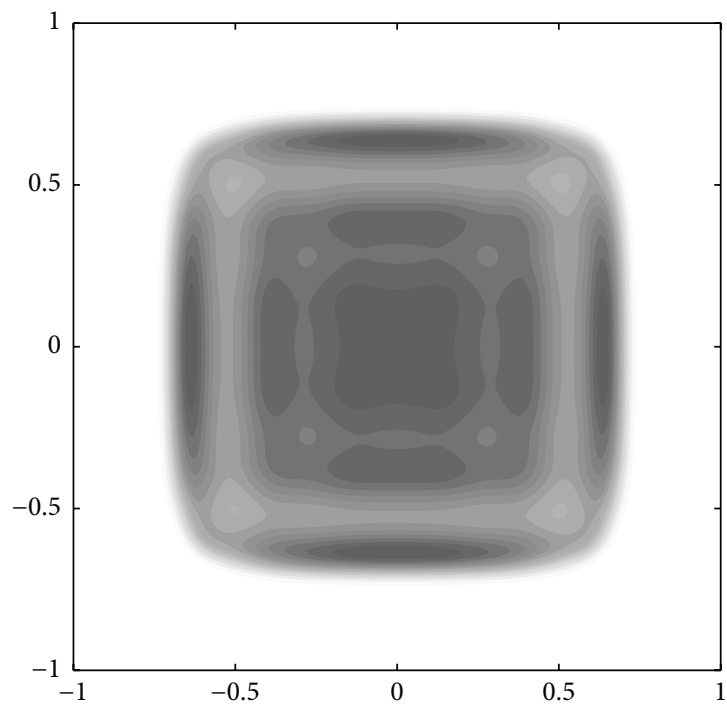

(e) Time $t=0.2, \sigma=100$

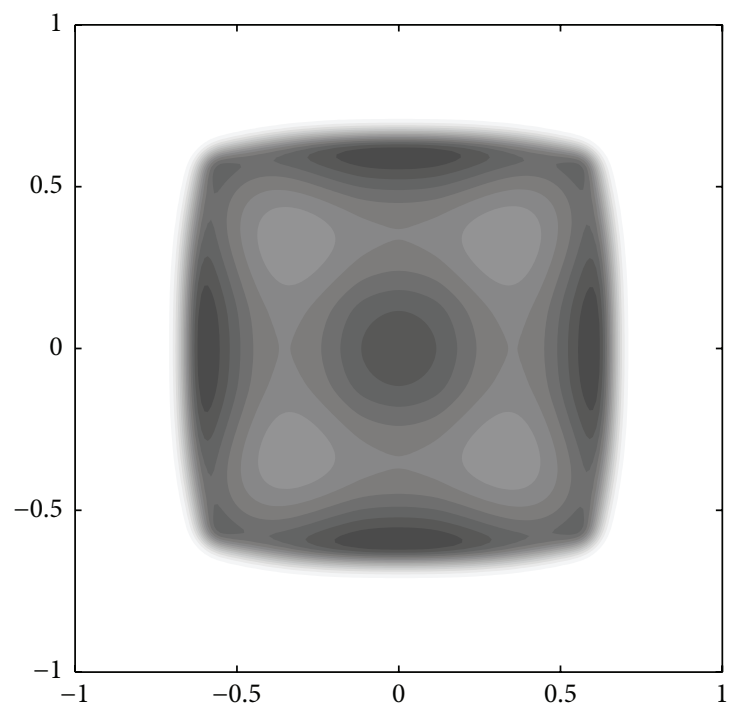

(b) Time $t=0.2, \sigma=25$

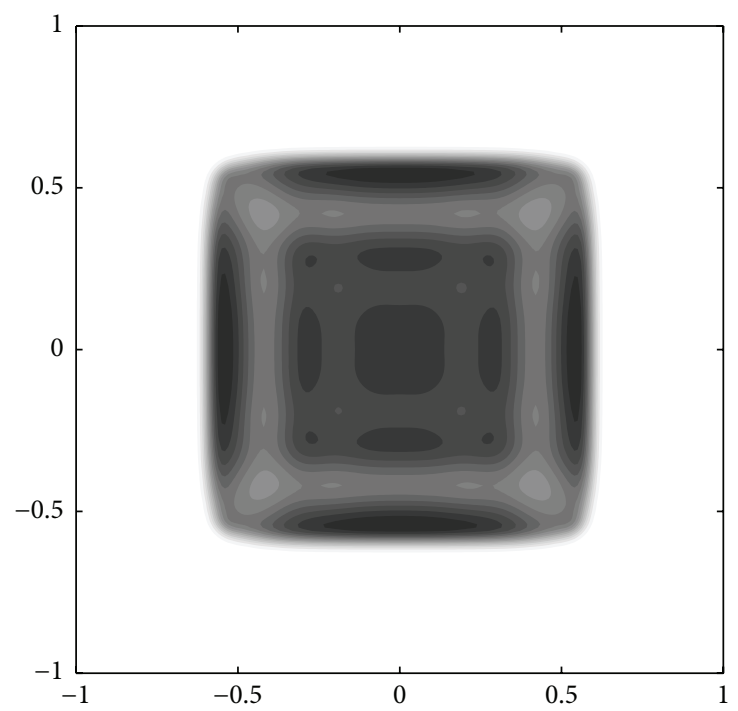

(d) Time $t=0.1, \sigma=100$

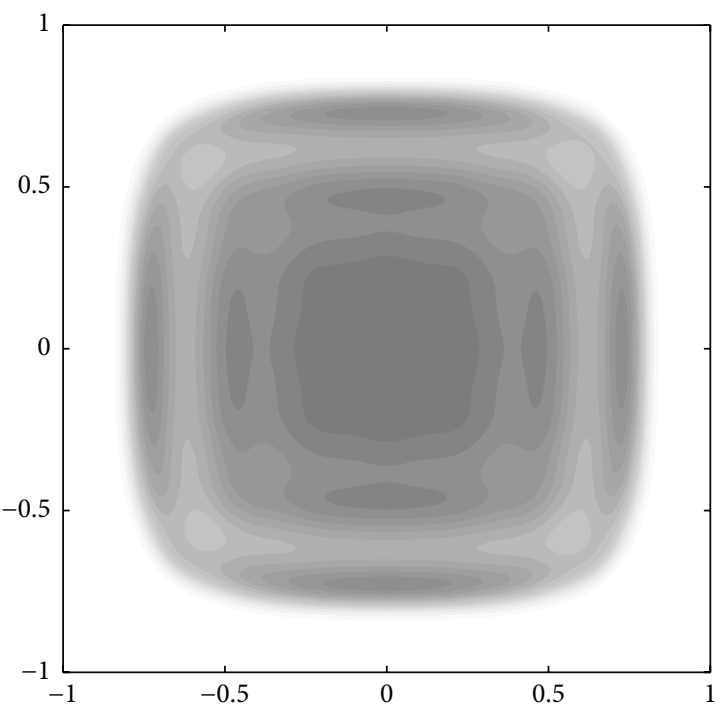

(f) Time $t=0.3, \sigma=100$

Figure 8: Continued. 


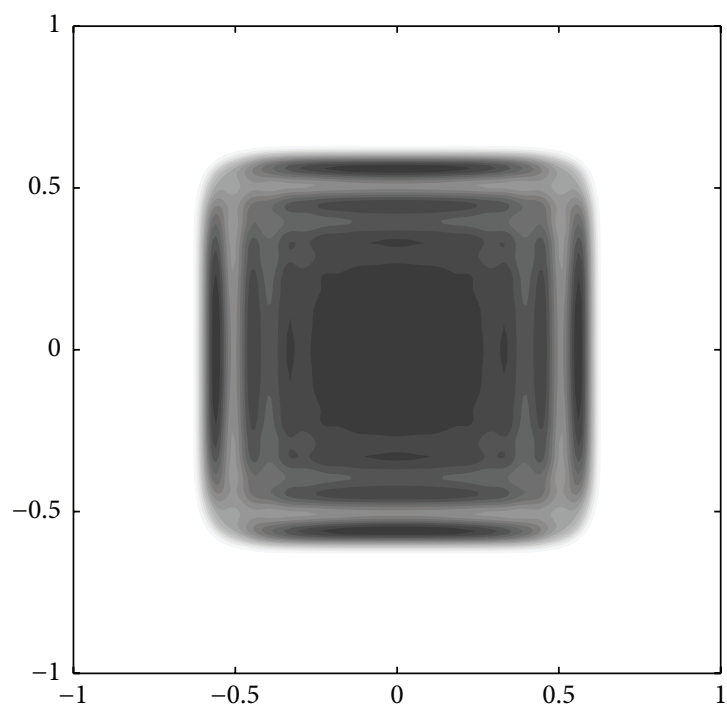

(g) Time $t=0.1, \sigma=400$

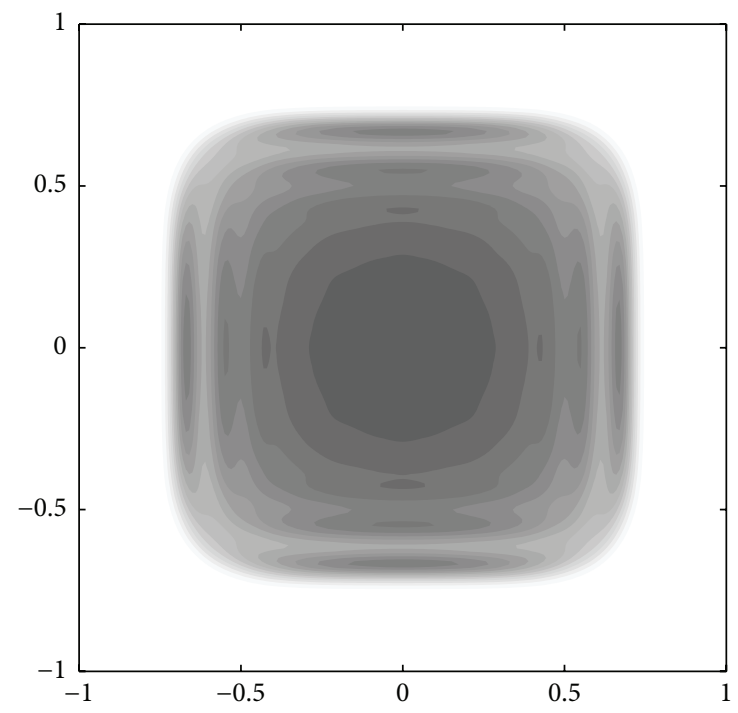

(h) Time $t=0.2, \sigma=400$

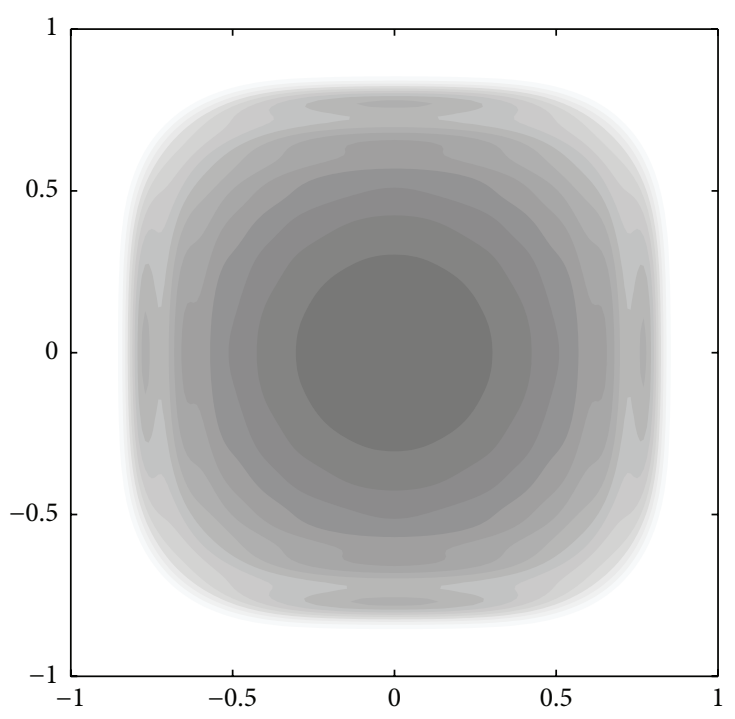

(i) Time $t=0.3, \sigma=400$

FIGURE 8: Numerical solution of the simplified model (41) computed by the finite volume approach with dimensional splitting. Visualized for times $t=0.1,0.2,0.3$ and smoothing function parameter $\sigma=25,100,400$.

All plots (exceptional (a) and (d)) lead to the same result and they are similar to the plot of Figure 8(e). Indeed, a low triangulation and a low polynomial degree cause poor results (cf. Figures 9(a) and 9(d)). To get the most solution accuracy, the usage of filters for the DG computations is neglected. Therefore, some high frequent oscillations can appear (cf. Figure 8(c)).

\section{Conclusion}

We have presented a novel numerical simulation algorithm, the discontinuous Galerkin method, to compute the movement of material flow on conveyor belts. The numerical difficulties arise from the predefined geometry of the setting and the flux function consisting of a nonlocal term including a convolution. We have tested the performance of the discontinuous Galerkin method against a finite volume scheme and observed satisfactory results. In addition to the good qualitative behavior of the numerical results, we also detected and verified solution artifacts as lane formation in both numerical approaches.

\section{Conflict of Interests}

The authors declare that there is no conflict of interests regarding the publication of this paper. 


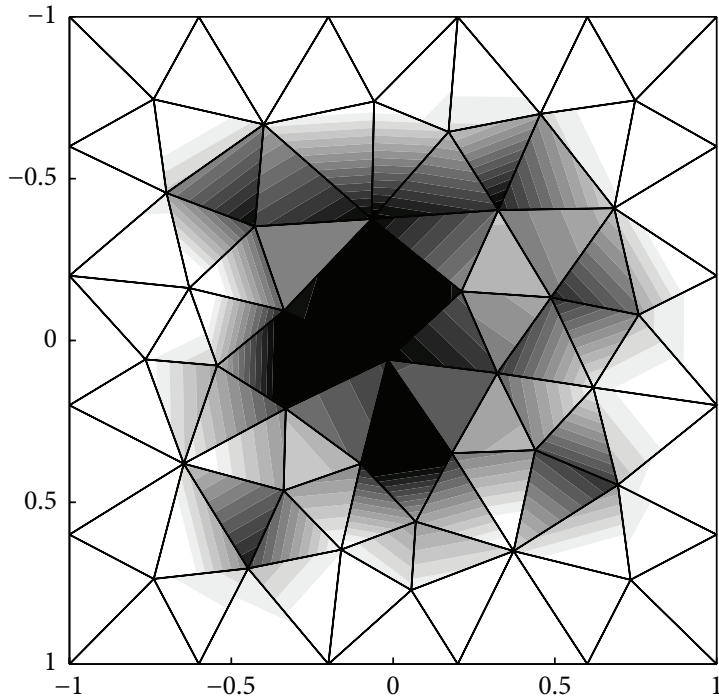

(a) $h \approx 0.4, N=1$

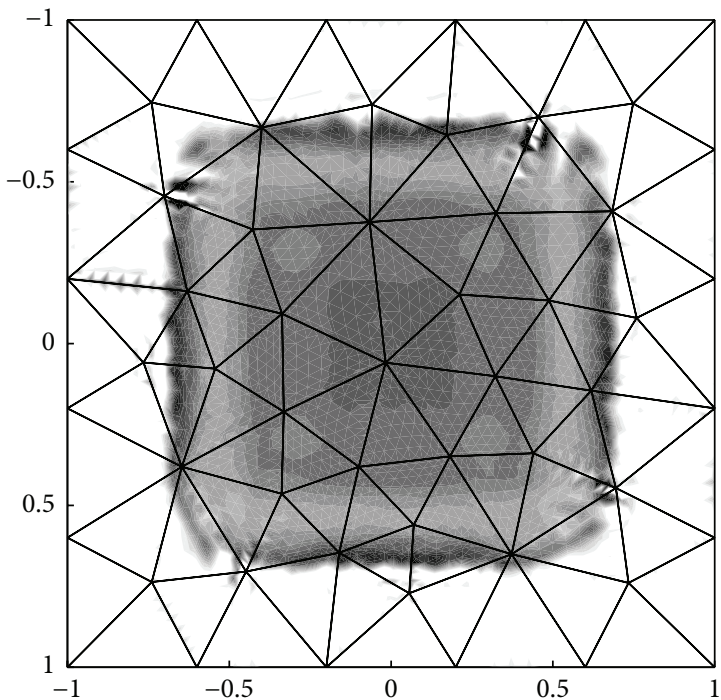

(c) $h \approx 0.4, N=11$

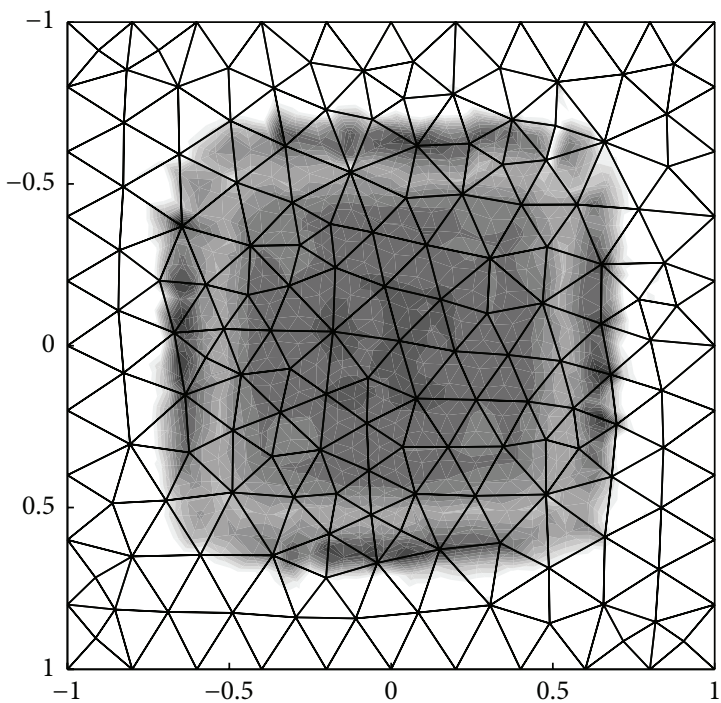

(e) $h \approx 0.2, N=3$

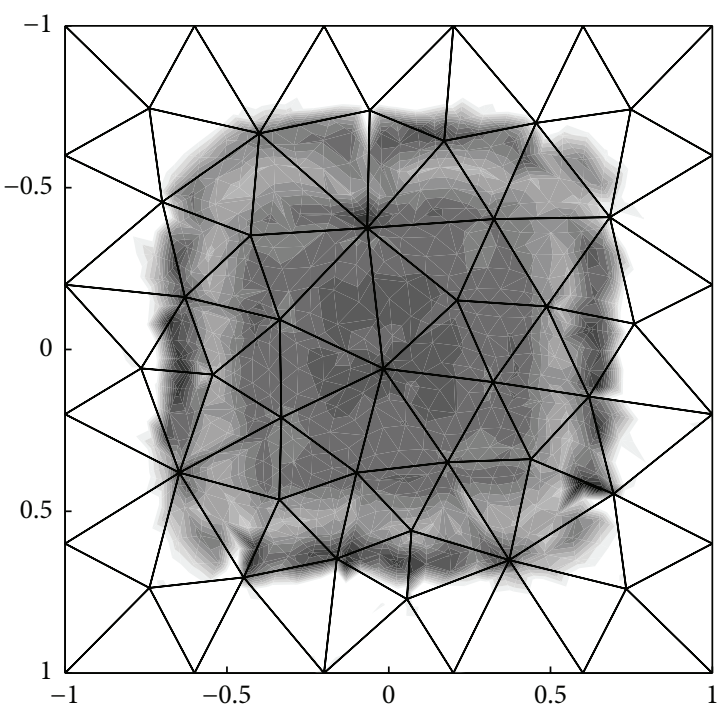

(b) $h \approx 0.4, N=5$

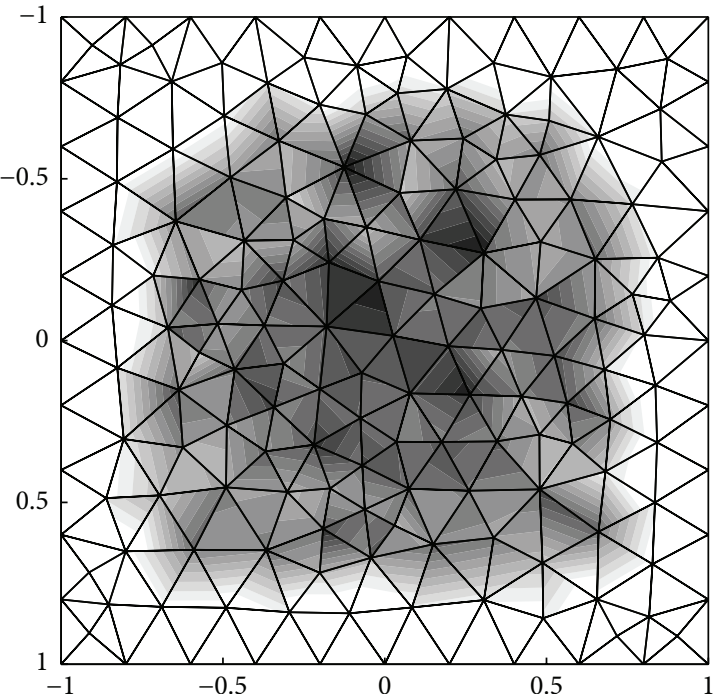

(d) $h \approx 0.2, N=1$

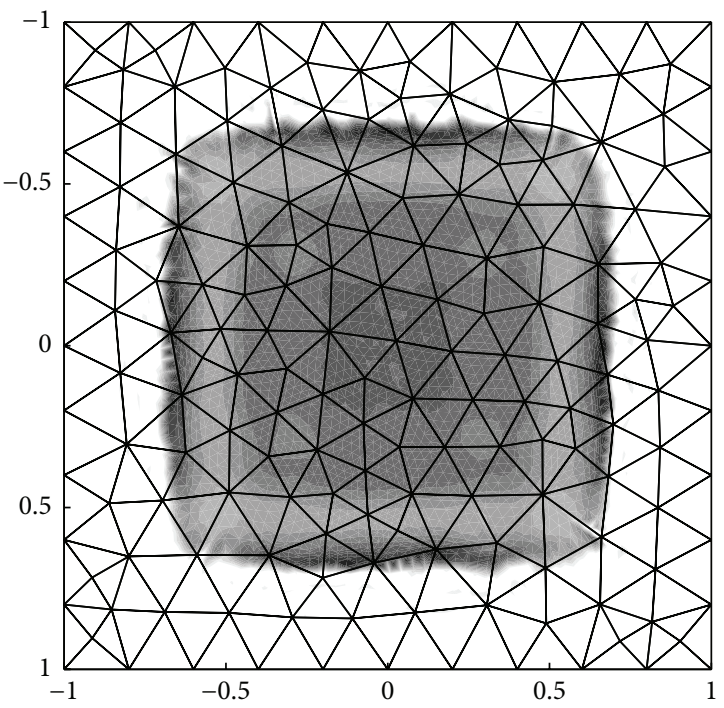

(f) $h \approx 0.2, N=7$

FIgURE 9: Continued. 


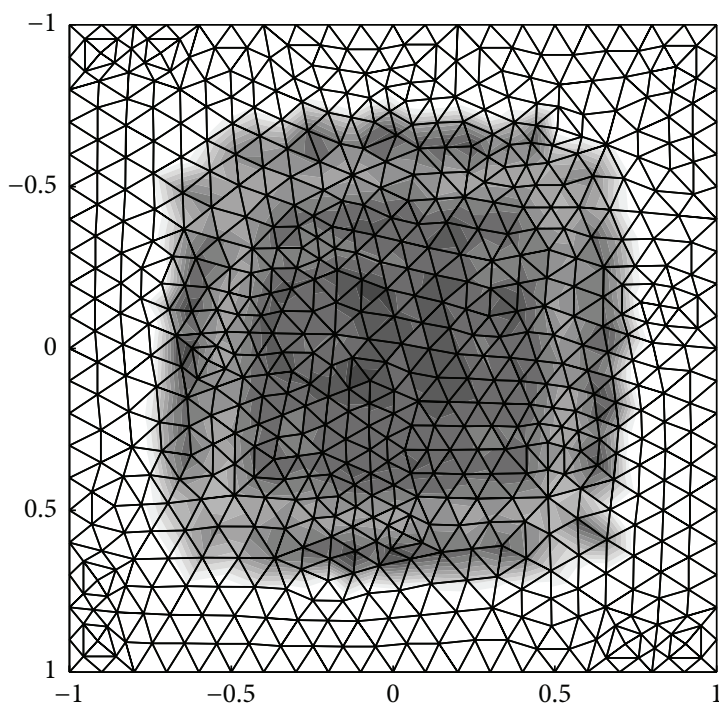

(g) $h \approx 0.1, N=1$

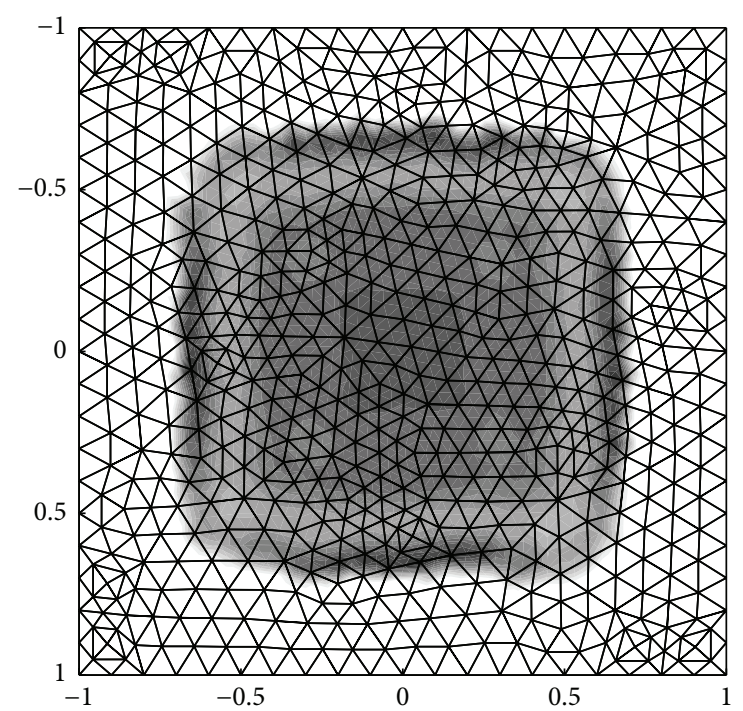

(h) $h \approx 0.1, N=2$

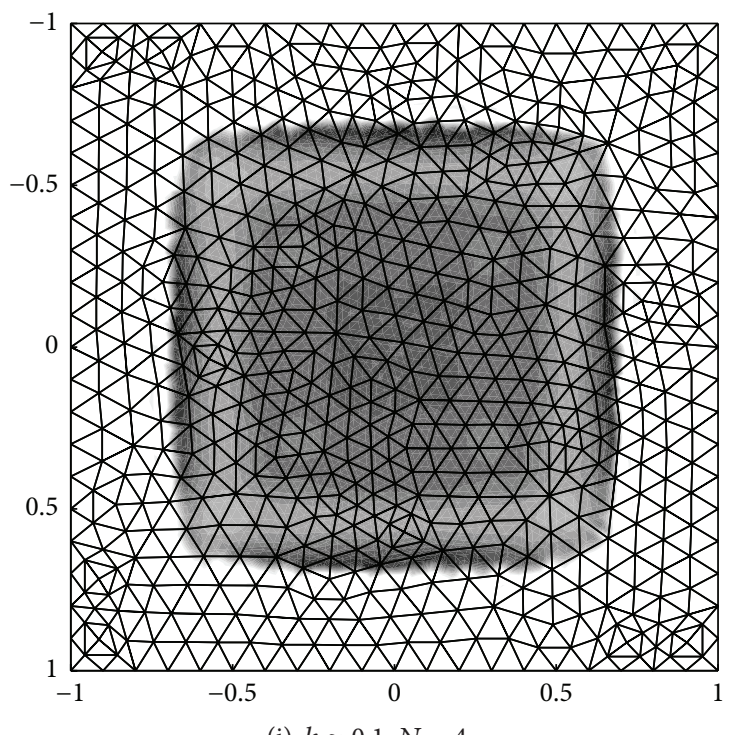

(i) $h \approx 0.1, N=4$

Figure 9: Comparison of the results of the discontinuous Galerkin method with different mesh-sizes $h$ and polynomial degrees $N$. The plots show the solution at time $t=0.2$ for a smoothness parameter $\sigma=100$.

\section{Acknowledgments}

This work was financially supported by the German Research Foundation (DFG), DFG grant OptiFlow (Project-ID GO 1920/3-1). Special thanks go to Veronika Schleper for fruitful and inspiring discussions.

\section{References}

[1] S. Hoher, P. Schindler, S. Göttlich, V. Schleper, and S. Röck, "System dynamic models and real-time simulation of complex material flow systems," in Enabling Manufacturing Competitiveness and Economic Sustainability, H. A. ElMaraghy, Ed., part 3, pp. 316-321, Springer, Berlin, Germany, 2012.

[2] G. Reinhart and F.-F. Lacour, "Physically based virtual commissioning of material flow intensive manufacturing plants," in
Procedings of the 3rd International Conference on Changeable, Agile, Reconfigurable and Virtual Production (CARV '09), H. A. Zaeh and M. F. ElMaraghy, Eds., pp. 377-387, Munich, Germany, 2009.

[3] S. Röck, "Hardware in the loop simulation of production systems dynamics," Production Engineering, vol. 5, no. 3, pp. 329-337, 2011.

[4] T. Gaugele, F. Fleissner, and P. Eberhard, "Simulation of material tests using meshfree Lagrangian particle methods," Proceedings of the Institution of Mechanical Engineers Part K: Journal of Multi-Body Dynamics, vol. 222, no. 4, pp. 327-338, 2008.

[5] S. Göttlich, S. Hoher, P. Schindler, V. Schleper, and A. Verl, "Modeling, simulation and validation of material flow on conveyor belts," Applied Mathematical Modelling, vol. 38, no. 13, pp. 3295-3313, 2014. 
[6] P. A. Langston, U. Tüzün, and D. M. Heyes, "Discrete element simulation of granular flow in 2D and 3D hoppers: dependence of discharge rate and wall stress on particle interactions," Chemical Engineering Science, vol. 50, no. 6, pp. 967-987, 1995.

[7] V. L. Popov, Contact Mechanics and Friction: Physical Principles and Applications, Springer, Berlin, Germany, 2010.

[8] H. P. Zhu and A. B. Yu, "Averaging method of granular materials," Physical Review E-Statistical, Nonlinear, and Soft Matter Physics, vol. 66, no. 2, Article ID 021302, 2002.

[9] H. P. Zhu and A. B. Yu, "Micromechanic modeling and analysis of unsteady-state granular flow in a cylindrical hopper," Journal of Engineering Mathematics, vol. 52, no. 1-3, pp. 307-320, 2005.

[10] M. Garavello and B. Piccoli, "Conservation laws models," in Traffic Flow on Networks, vol. 1 of AIMS Series on Applied Mathematics, American Institute of Mathematical Sciences (AIMS), Springfield, Ill, USA, 2006.

[11] C. D’Apice, S. Göttlich, M. Herty, and B. Piccoli, Modeling, Simulation, and Optimization of Supply Chains: A Continuous Approach, Society for Industrial and Applied Mathematics, Philadelphia, Pa, USA, 2010.

[12] R. M. Colombo, M. Garavello, and M. Lécureux-Mercier, "A class of nonlocal models for pedestrian traffic," Mathematical Models and Methods in Applied Sciences, vol. 22, no. 4, Article ID 1150023, 2012.

[13] R. M. Colombo and M. Lécureux-Mercier, "Nonlocal crowd dynamics models for several populations," Acta Mathematica Scientia-Series B: English Edition, vol. 32, no. 1, pp. 177-196, 2012.

[14] J. H. Evers, S. C. Hille, and A. Muntean, "Mild solutions to a measure-valued mass evolution problem with flux boundary conditions," Journal of Differential Equations, vol. 259, no. 3, pp. 1068-1097, 2015.

[15] A. Aggarwal, R. M. Colombo, and P. Goatin, "Nonlocal systems of conservation laws in several space dimensions," SIAM Journal on Numerical Analysis, vol. 53, no. 2, pp. 963-983, 2015.

[16] S. Göttlich, A. Klar, and S. Tiwari, "Complex material flow problems: a multi-scale model hierarchy and particle methods," Journal of Engineering Mathematics, vol. 92, pp. 15-29, 2015.

[17] S. Canic, B. Piccoli, J.-M. Qiu, and T. Ren, "Runge-Kutta discontinuous Galerkin method for traffic flow model on networks," Journal of Scientific Computing, vol. 63, no. 1, pp. 233-255, 2015.

[18] B. Cockburn and C. W. Shu, "The Runge-Kutta discontinuous Galerkin method for conservation laws V: multidimensional systems," Journal of Computational Physics, vol. 141, no. 2, pp. 199-224, 1998.

[19] V. Gowda and J. Jaffré, "A discontinuous finite element method for scalar nonlinear conservation laws," Rapport de Recherche INRIA, Institut National de Recherche en Informatique et en Automatique, Rocquencourt, France, 1993.

[20] J. S. Hesthaven and T. Warburton, Nodal Discontinuous Galerkin Methods-Algorithms, Analysis, and Applications, Springer, Berlin, Germany, 2008.

[21] H. Hoteit, P. Ackerer, R. Mosé, J. Erhel, and B. Philippe, "New two-dimensional slope limiters for discontinuous Galerkin methods on arbitrary meshes," International Journal for Numerical Methods in Engineering, vol. 61, no. 14, pp. 2566-2593, 2004.

[22] S. Sun and M. Dong, "Continuum modeling of supply chain networks using discontinuous Galerkin methods," Computer Methods in Applied Mechanics and Engineering, vol. 197, no. 1316, pp. 1204-1218, 2008.
[23] S. Zhang, S. Sun, and H. Yang, "Optimal convergence of discontinuous Galerkin methods for continuum modeling of supply chain networks," Computers \& Mathematics with Applications, vol. 68, no. 6, pp. 681-691, 2014.

[24] R. J. LeVeque, Finite Volume Methods for Hyperbolic Problems, Cambridge Texts in Applied Mathematics, Cambridge University Press, Cambridge, UK, 2002.

[25] F. Bassi and S. Rebay, "High-order accurate discontinuous finite element solution of the 2D Euler equations," Journal of Computational Physics, vol. 138, no. 2, pp. 251-285, 1997.

[26] B. J. Block, M. Lukáčová-Medvid’ová, P. Virnau, and L. Yelash, "Accelerated GPU simulation of compressible flow by the discontinuous evolution Galerkin method," European Physical Journal: Special Topics, vol. 210, no. 1, pp. 119-132, 2012.

[27] B. Cockburn, G. Karniadakis, and C. Shu, Discontinuous Galerkin Methods. Theory, Computation and Applications, Lecture Notes in Computational Science and Engineering, Springer, 2000.

[28] D. Gottlieb and C.-W. Shu, "On the Gibbs phenomenon and its resolution," SIAM Review, vol. 39, no. 4, pp. 644-668, 1997.

[29] C. Canuto, M. Y. Hussaini, A. Quarteroni, and T. A. Zang, Spectral Methods in Fluid Dynamics, Springer Series in Computational Physics, Springer, Berlin, Germany, 1988.

[30] J. S. Hesthaven and R. M. Kirby, "Filtering in legendre spectral methods," Mathematics of Computation, vol. 77, no. 263, pp. 1425-1452, 2008. 


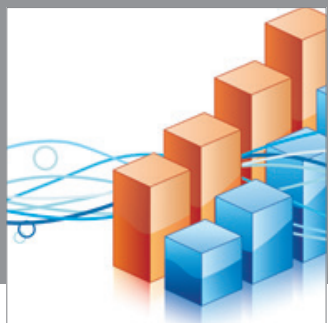

Advances in

Operations Research

mansans

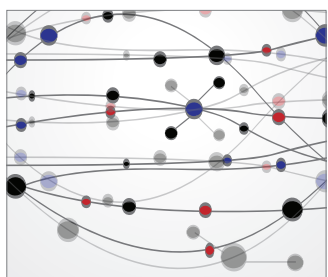

The Scientific World Journal
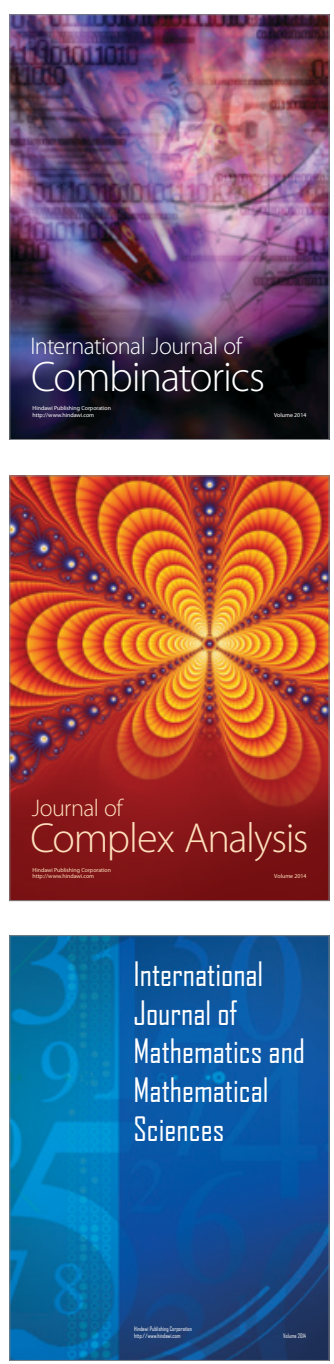
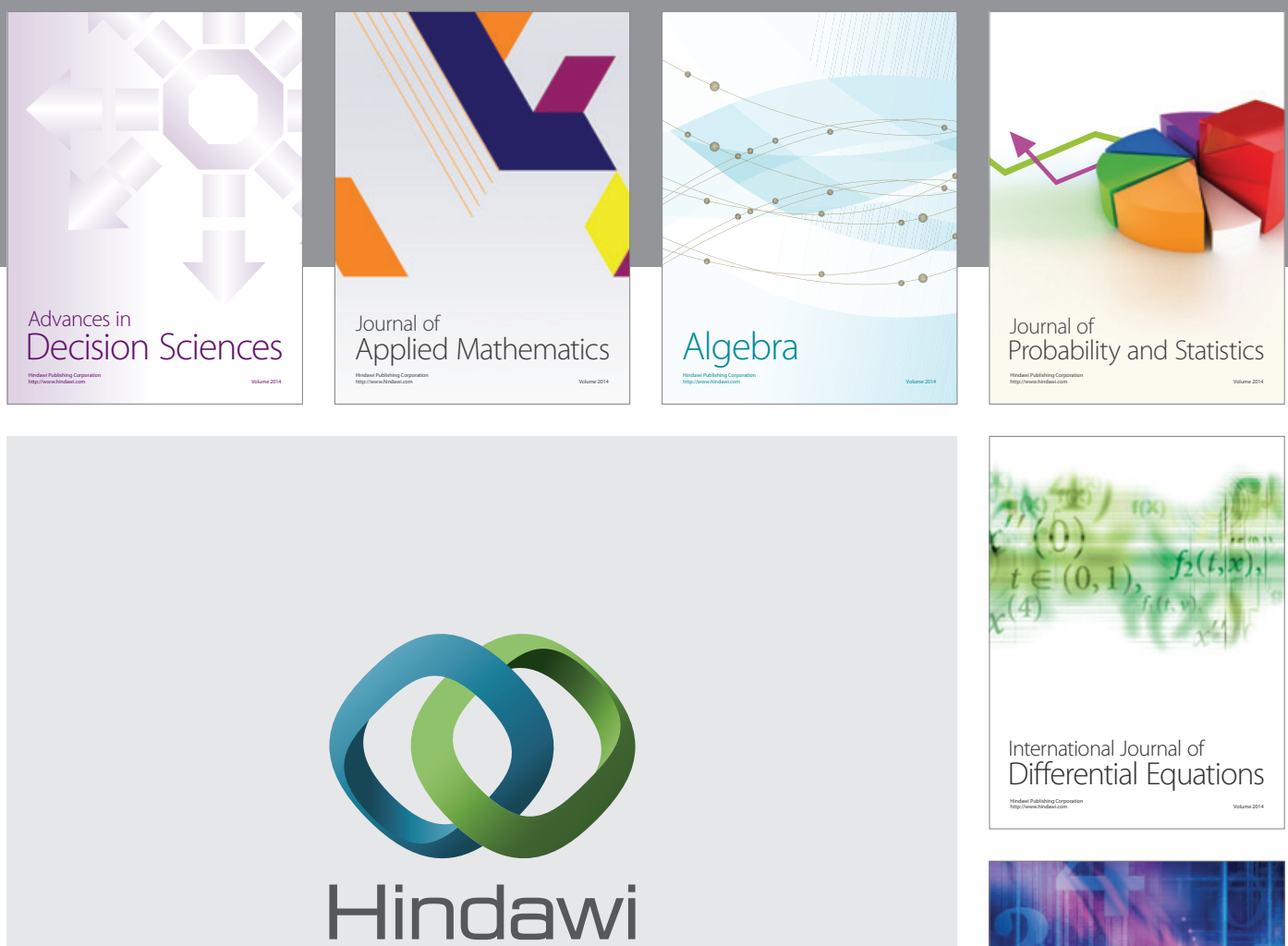

Submit your manuscripts at http://www.hindawi.com
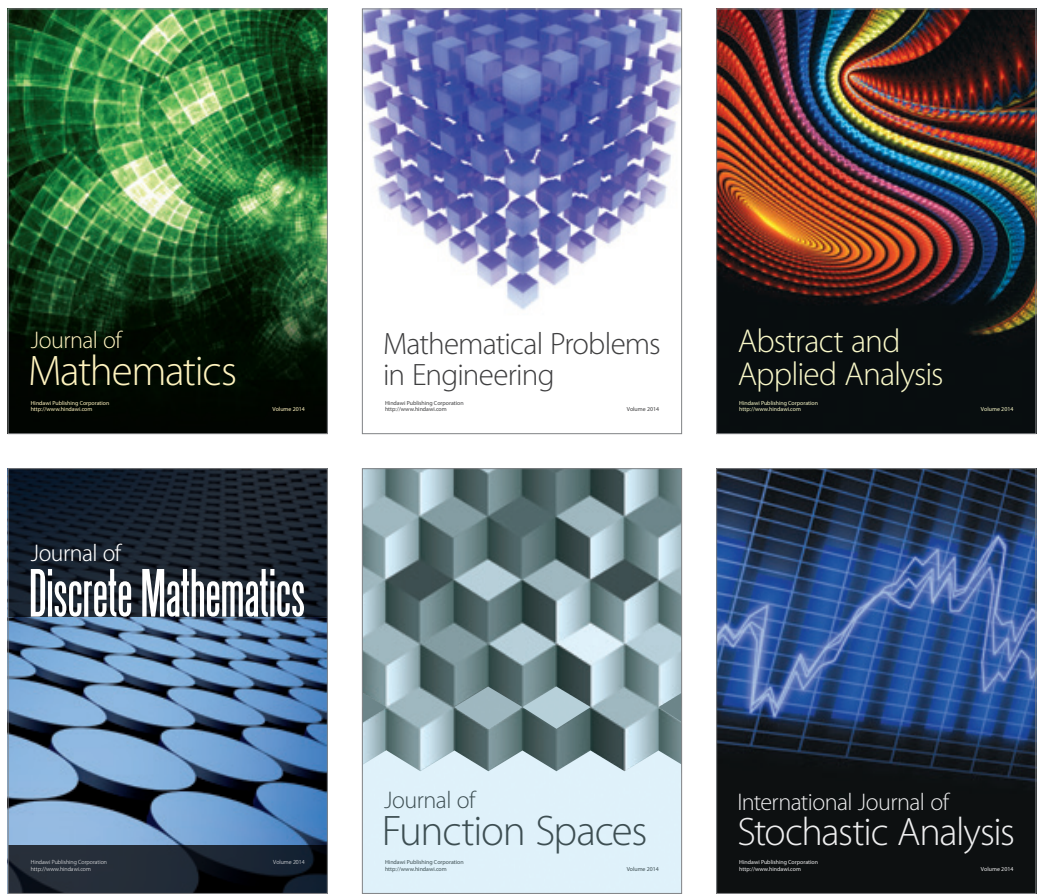

Journal of

Function Spaces

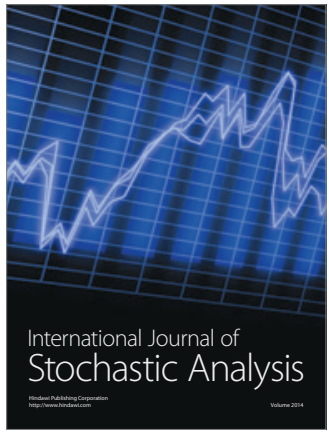

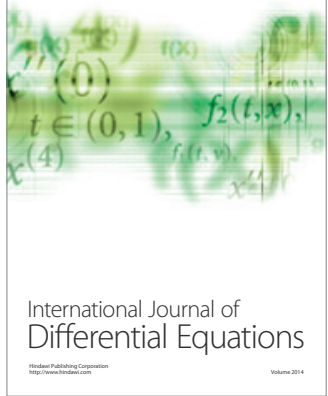
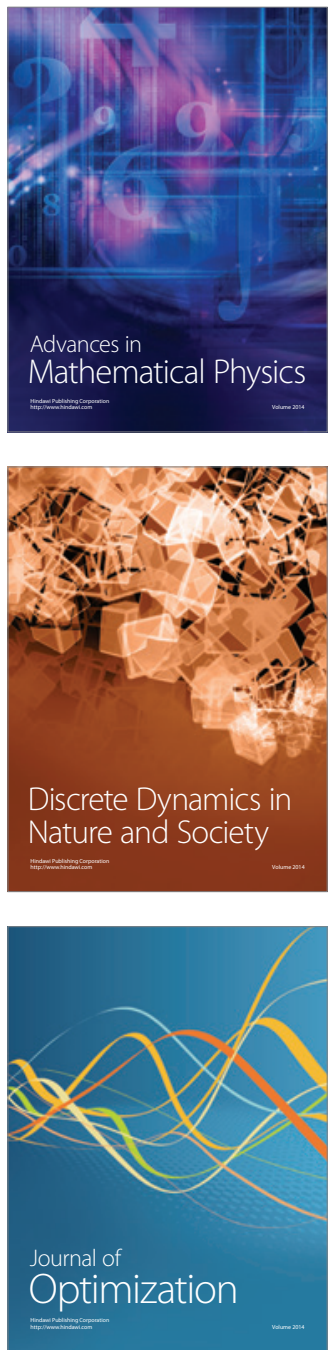